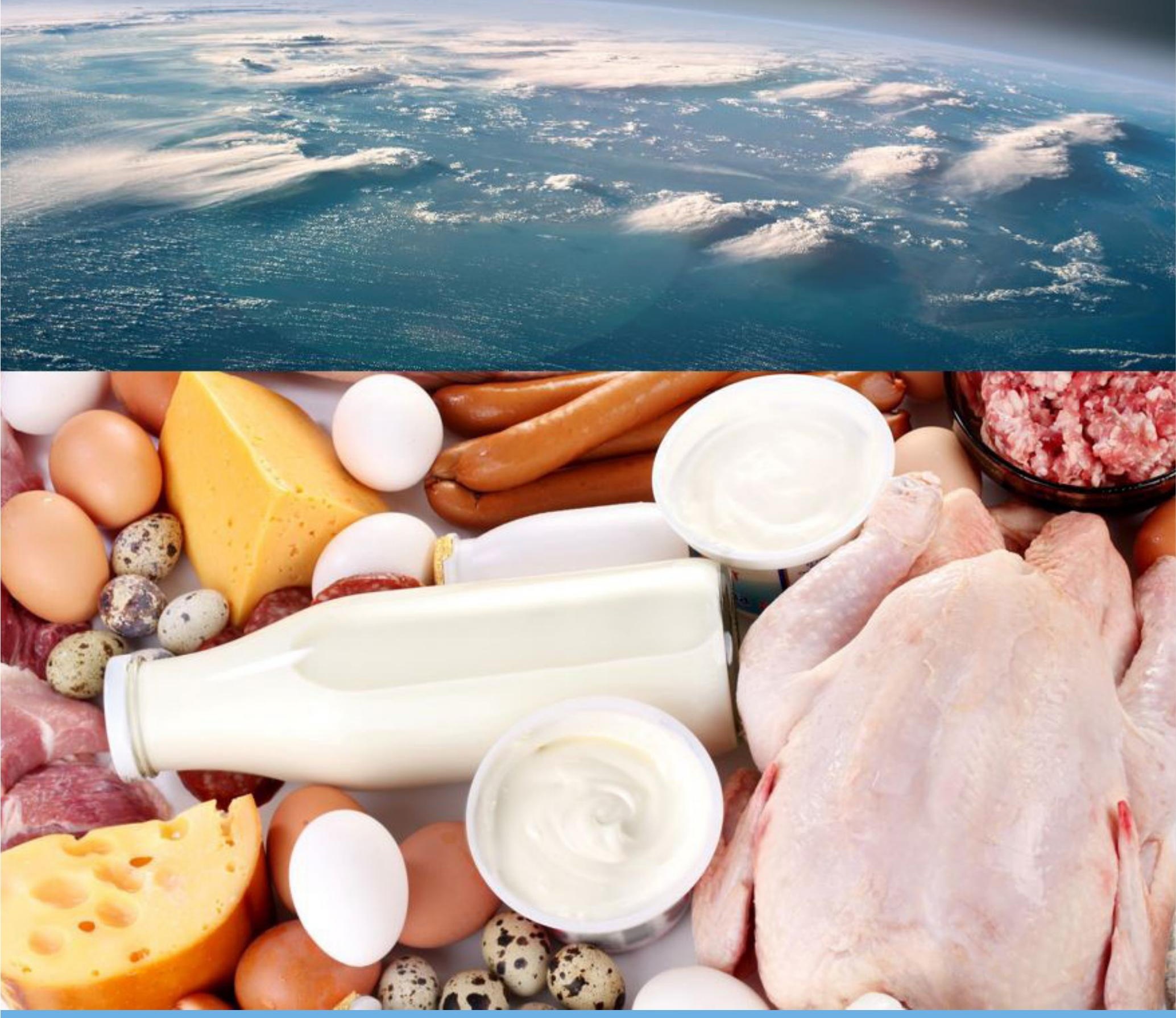

The contribution of breeding to reducing environmental impact of animal production 



\section{The contribution of breeding to reducing environmental impact of animal production}

H. Mollenhorst and Y. de Haas

Animal Breeding and Genomics

This research was conducted by Wageningen Livestock Research, financially supported by the Dutch Ministry of Economic Affairs (TKI Agri \& Food project 16022) and the Breed4Food partners Cobb Europe, CRV, Hendrix Genetics and Topigs Norsvin.

Wageningen Livestock Research

Wageningen, March 2019

Report 1156 
Mollenhorst, H., Y. de Haas, 2019. The contribution of breeding to reducing environmental impact of animal production. Wageningen Livestock Research, Report 1156.

Samenvatting De veehouderij is wereldwijd verantwoordelijk voor $14,5 \%$ van de totale antropogene (door de mens veroorzaakte) broeikasgasemissies. Ongeveer de helft van deze emissies komt rechtstreeks vanuit de veehouderij, terwijl de andere helft zijn oorsprong vindt in de voerproductie. De fokkerij heeft als doel om de veehouderij te verbeteren en een efficiënt gebruik van grondstoffen te bevorderen, waardoor de milieubelasting af zal nemen. Het doel van het in dit rapport beschreven onderzoek was om de bijdrage van fokkerij aan het verminderen van de milieubelasting door de vier belangrijkste diersoorten in de Nederlandse veehouderij (met hun producten) te berekenen, namelijk kippen (vlees), legkippen (eieren), varkens (vlees) en koeien (melk). Het onderzoek is gedaan middels een combinatie van een literatuurstudie en een kwantitatieve analyse om de huidige milieubelasting en de gevolgen van recente fokkerij gerelateerde ontwikkelingen in te schatten. Voor kippenvlees, eieren en varkensvlees lag hierbij de focus op broeikasgasemissies en stikstof- en fosfaatefficiëntie, terwijl bij melk gefocust is op methaanemissie vanuit de koe. Methaan is een belangrijk broeikasgas. De resultaten van dit onderzoek geven aan dat door fokkerij de milieubelasting van dierlijke producten met ongeveer $1 \%$ per jaar daalt. Dit wordt behaald zonder specifiek op milieukenmerken te selecteren, maar is vooral een gevolg van selectie op (voer-)efficiëntie.

Summary Animal production is responsible for $14.5 \%$ of total anthropogenic greenhouse gas (GHG) emissions. Approximately half of these emissions originate directly from animal production, whereas the other half comes from feed production. Animal breeding aims at improving animal production and efficient use of resources, which results in a reduction of environmental impacts. The objective of this study was to quantify the contribution of animal breeding to reducing the environmental impact of the four major livestock species in the Netherlands (with their animal product), namely broilers (meat), laying hens (eggs), pigs (meat) and dairy cattle (milk). This study comprised of a literature review and a quantitative assessment of the current environmental impact and the result of recent genetic improvements. For broiler meat, chicken eggs and pig meat the focus was laid on GHG emissions and nitrogen and phosphorus efficiency, whereas for dairy the focus was laid on enteric methane emissions, an important contributor to GHG emissions. Results show that breeding reduces environmental impacts of animal products by about $1 \%$ per year. This is achieved without specific selection on environmental traits, but as an indirect response through selection on increased (feed) efficiency.

This report can be downloaded for free at https://doi.org/10.18174/472395 or at www.wur.nl/livestock-research (under Wageningen Livestock Research publications).

\section{(C) 2019 Wageningen Livestock Research}

P.O. Box 338, $6700 \mathrm{AH}$ Wageningen, The Netherlands, T +31 (0)317 483953 ,

E info.livestockresearch@wur.nl, www.wur.nl/livestock-research. Wageningen Livestock Research is part of Wageningen University \& Research.

All rights reserved. No part of this publication may be reproduced and/or made public, whether by print, photocopy, microfilm or any other means, without the prior permission of the publisher or author.

Wageningen Livestock Research is NEN-EN-ISO 9001:2015 certified.

All our research commissions are in line with the Terms and Conditions of the Animal Sciences Group. These are filed with the District Court of Zwolle. 


\section{Table of contents}

$\begin{array}{ll}\text { Foreword } & \mathbf{5}\end{array}$

$\begin{array}{ll}\text { Executive summary } & 7\end{array}$

1

$\begin{array}{ll}\text { Introduction and outline } & 9\end{array}$

2

$\begin{array}{ll}\text { Literature review } & 12\end{array}$

$2.1 \quad$ Environmental impact of different species 12

2.2 Historical trends - Broilers 13

2.3 Historical trends - Layers 16

2.4 Historical trends - Pigs $\quad 19$

2.5 Historical trends - Dairy 21

2.6 Conclusion and Discussion literature review 23

3 Quantification of contribution of animal breeding 25

3.1 Quantification - Broilers 26

$\begin{array}{lll}3.2 & \text { Quantification - Layers } & 30\end{array}$

$\begin{array}{lll}3.3 & \text { Quantification - Pigs } & 37\end{array}$

$\begin{array}{lll}3.4 & \text { Quantification - Dairy } & 41\end{array}$

4

$\begin{array}{ll}\text { Conclusions and recommendations } & 45\end{array}$

$\begin{array}{ll}\text { References } & 46\end{array}$ 



\section{Foreword}

Livestock has always had an important role in the global food production. Over many years, Breed4Food partners have successfully selected for animals that efficiently produce meat, eggs and dairy. At the same time, however, the environmental impact has become an important sustainability issue. Topical examples include the contribution to global warming by the emission of greenhouse gases and the depletion of scarce resources such as phosphorus. Current and future challenges in the breeding sector are therefore to adequately respond to the growing demand for animal protein whilst also reducing its environmental impact.

The Breed4Food consortium invests in pre-competitive research to contribute to sustainable animal production. The current report evaluates the environmental impact of past breeding strategies and discusses future developments. The study is the result of a successful collaboration between WUR and leading breeding companies. I thank the authors and everyone who participated in the discussions and I am confident that this report will be an important step towards a further optimised role of animal breeding in a sustainable livestock production.

Erwin Koenen

Breed4Food Director 


\section{Executive summary}

Animal production is responsible for $14.5 \%$ of total anthropogenic greenhouse gas (GHG) emissions. Approximately half of these emissions originate directly from animal production, whereas the other half comes from feed production. Animal breeding aims at improving animal production and efficient use of resources, which results in a reduction of the environmental impact. The objective of this study was to quantify the contribution of animal breeding to reducing the environmental impact of the four major livestock species in the Netherlands (with their animal product), namely broilers (meat), laying hens (eggs), pigs (meat) and dairy cattle (milk).

A literature review was performed to assess the current status of and historical trends in environmental impact, mainly focussed on GHG emissions, based on general performance criteria. Emissions related to feed production dominate impacts of broilers, laying hens and, to a minor extent, pigs. For dairy cattle, enteric methane emission is a large contributor to total GHG emissions. Historical trends show considerable improvements in efficiency over the last decades, in which breeding plays an important role. From the literature review we concluded that the contribution of breeding to reducing environmental impact of animal production is led by an indirect response through selection on increased efficiency.

Next to the literature review, a quantitative assessment was made on the current environmental impact of the four animal products and the effect of recent genetic improvements. For broiler meat, chicken eggs and pig meat the focus was laid on GHG emissions and nitrogen and phosphorus efficiency, whereas for dairy the focus was laid on enteric methane emissions, an important contributor to GHG emissions. Data were partly provided by breeding organisations, partners in the Breed4Food consortium. In general, results showed that breeding reduces environmental impacts of animal products by about $1 \%$ per year.

- For laying hens, white and brown hens were considered and it was concluded that white hens have a lower GHG impact and better $\mathrm{N}$ and $\mathrm{P}$ efficiency than brown hens and that improvements over the past 10 years went faster for white hens as well.

- For broilers it was shown that GHG emissions decreased and $\mathrm{N}$ and $\mathrm{P}$ efficiency increased with more than $1 \%$. However, only data of a 4-years' timeframe under less controlled circumstances were available, which resulted in a possible overestimation of genetic progress.

- For pigs data were available from a well-controlled study with two diets and animals divided by sex; however, the time frame was only two years. Results showed that also for pigs in the growingfattening phase, GHG emissions decrease and N and P efficiency increase with the current breeding goal. Furthermore, boars had lower environmental impact than gilts.

- For dairy cattle, results showed that with the current breeding goal, methane production per cow per day increases, but methane intensity (i.e., methane production per kg milk) decreases.

All reported results are achieved without specific selection on environmental traits, but as an indirect response of the current breeding goals for each species, which is a combination of health, growth, and (feed) efficiency. If it is desired to select directly on environmental traits, recording of new traits is required, e.g., nitrogen and phosphorus contents of meat and eggs and methane emission of individual dairy cows.

Results of this study are reported in an extensive presentation that is digitally available through the authors or Breed4Food partners. A printed version of the presentation forms the core of this report. 


\section{Introduction and outline}

The contribution of breeding to reducing environmental impact of animal production

B4F Societal impact of environmental traits

Erwin Mollenhorst \& Yvette de Haas, Animal Breeding and Genomics
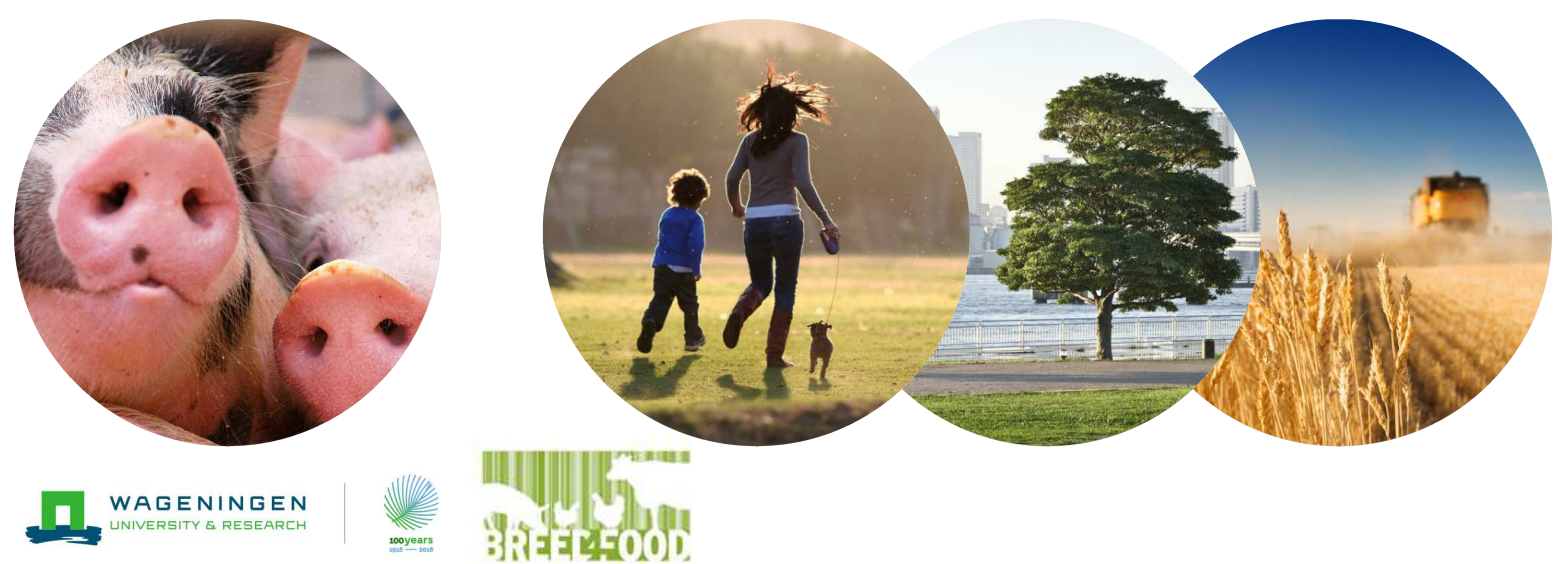

\section{Environmental impacts}

Most studied environmental impacts:

- Emission of greenhouse gases (GHG)

- $\mathrm{CO}_{2}, \mathrm{CH}_{4}$ and $\mathrm{N}_{2} \mathrm{O}$ summed as $\mathrm{CO}_{2}$-equivalents

- Efficient use of polluting and scarce mineral resources

- Nitrogen (N)

- Phosphorus (P) 


\section{Assessment methods}

- Life Cycle Assessment (LCA)

- Cradle to farm gate, i.e. including all inputs and emissions in preceding processes, like fertilizer and energy for feed production, production of young stock, etc.

- Nutrient use efficiencies

- Input over output calculation for specific production process

- General performance metrics

- Growth, production, etc.

- Feed conversion ratio (FCR; reciprocal of feed efficiency)

\section{Selection index}

- The national breeding goal for dairy in the Netherlands consists of several traits, related to production, conformation, health, fertility, calving ease and efficiency. These traits are weighted in an index to achieve optimal improvement of all traits in the desired directions

- In this study we assessed what will change when environmental impact trait is included 


\section{Outline}

Literature review

GHG emissions of different species

Historical trends (LCA / performance)

Quantification of contribution of animal breeding, per species

Conclusions

Recommendations 


\section{Literature review}

\subsection{Environmental impact of different species}

\section{GHG - Global perspective}

- Livestock total 7.1 Gton

(14.5\% of the total anthropogenic emissions)

- Beef

2.9 Gton

- Cattle milk

1.4 Gton

- Pig meat

0.7 Gton

- Chicken meat

0.4 Gton

- Chicken eggs

0.2 Gton

- Others

1.5 Gton

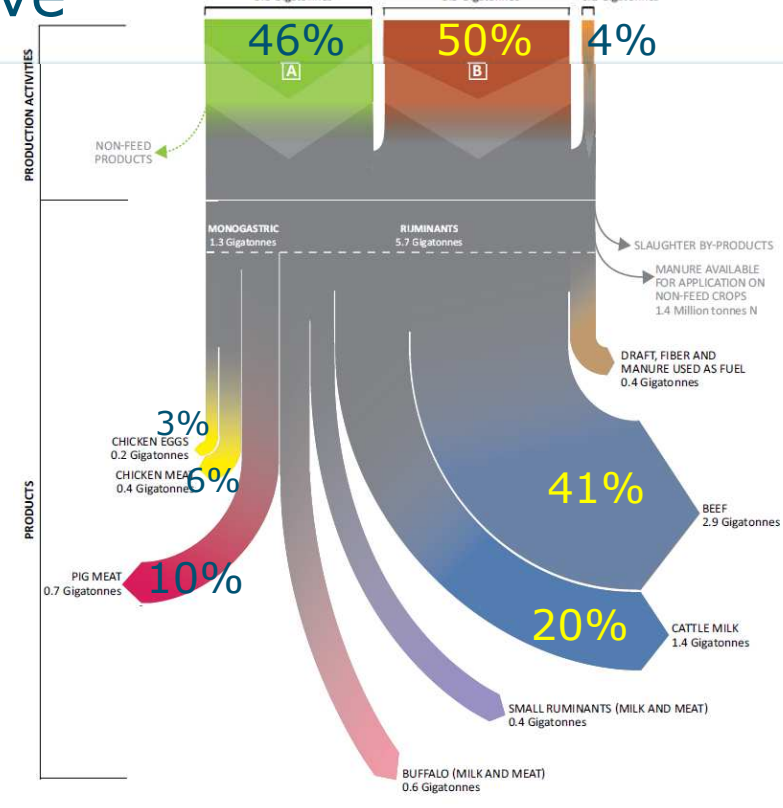

Source: FAO: Gerber et al, 2013

\section{GHG emissions of different species}
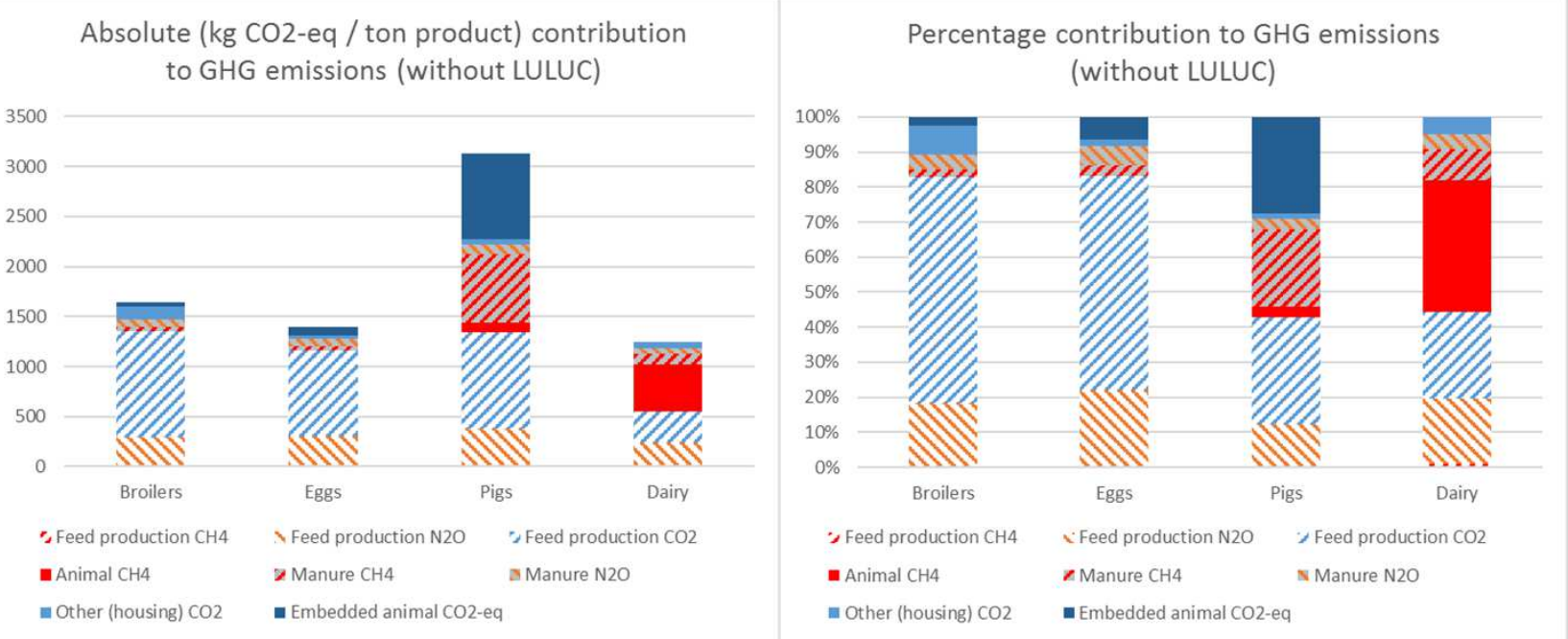
2.2 Historical trends - Broilers

\section{Broilers - GHG emissions}

Absolute ( $\mathrm{kg} \mathrm{CO} 2-\mathrm{eq} /$ ton product) contribution to GHG emissions (without LULUC)

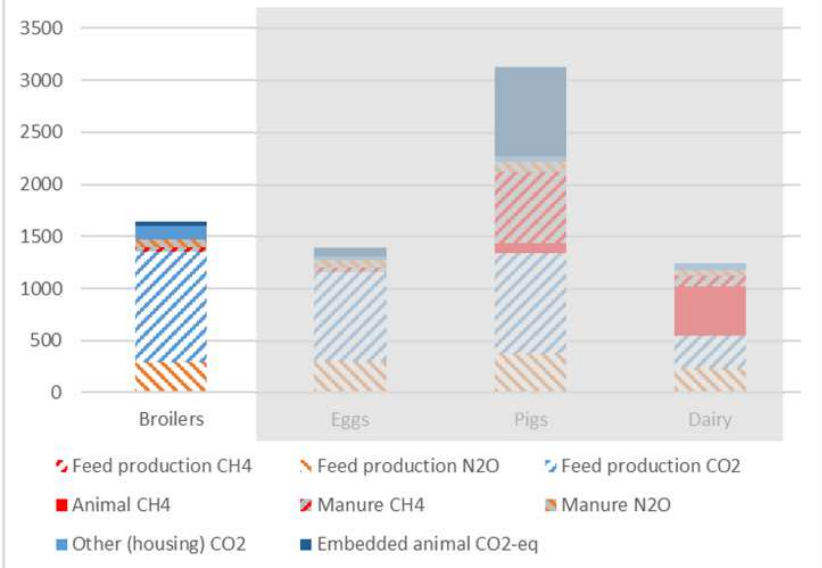

- $83 \%$ feed production

- $65 \% \mathrm{CO}_{2}$

- $18 \% \mathrm{~N}_{2} \mathrm{O}$

- $8 \%$ housing $\left(\mathrm{CO}_{2}\right)$

- $7 \%$ manure $\left(\mathrm{CH}_{4} / \mathrm{N}_{2} \mathrm{O}\right)$

- $2 \%$ reproduction

\section{Broilers - performance (1957 - 2001)}

Conclusions Havenstein et al.:

- $85-90 \%$ genetic selection

- $10-15 \%$ nutrition

Comparison at $1.8 \mathrm{~kg} \mathrm{BW}$ at corresponding diet

\begin{tabular}{|l|c|c|}
\hline $\mathbf{1 . 8}$ kg & 1957 & $\begin{array}{c}2001 \\
\text { BW }\end{array}$ \\
\hline ACRBC & Ross 308 \\
\hline FCR & 101 & 32 \\
\hline
\end{tabular}

\begin{tabular}{|l|c|c|}
\hline $\begin{array}{l}\text { 42-day } \\
\text { BW (kg) }\end{array}$ & $\begin{array}{c}1957 \\
\text { ACRBC }\end{array}$ & $\begin{array}{c}2001 \\
\text { Ross 308 }\end{array}$ \\
\hline 1957 diet & 0.54 & 2.13 \\
\hline 2001 diet & 0.58 & 2.67 \\
\hline
\end{tabular}

\begin{tabular}{|l|c|c|}
\hline $\begin{array}{l}\text { 42-day } \\
\text { FCR }\end{array}$ & $\begin{array}{c}1957 \\
\text { ACRBC }\end{array}$ & $\begin{array}{c}2001 \\
\text { Ross 308 }\end{array}$ \\
\hline 1957 diet & 2.34 & 1.92 \\
\hline 2001 diet & 2.14 & 1.63 \\
\hline
\end{tabular}




\section{Broilers - feed conversion (1970 - 2012)}

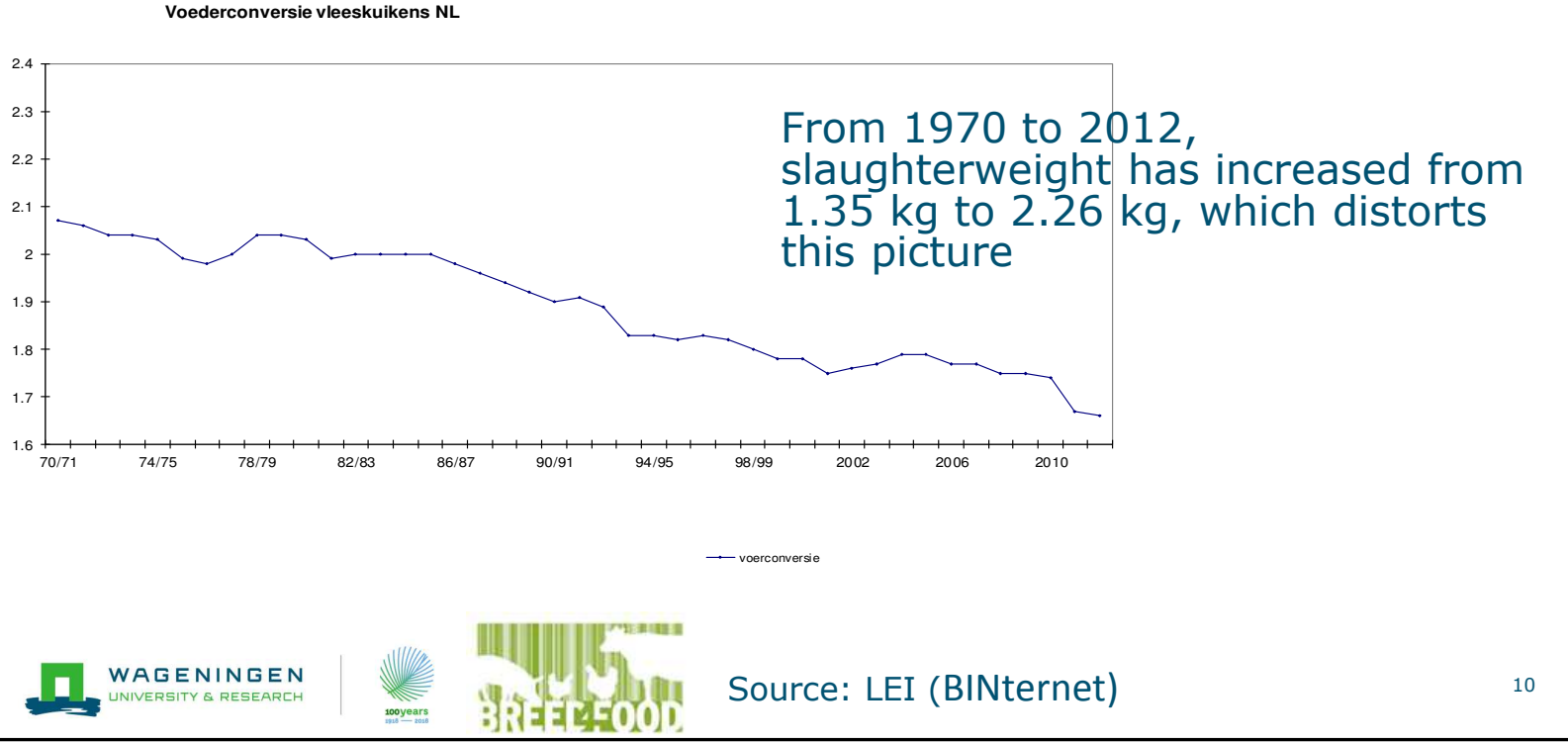

\section{Broilers - feed conversion (corrected to $2.15 \mathrm{~kg}$ )}

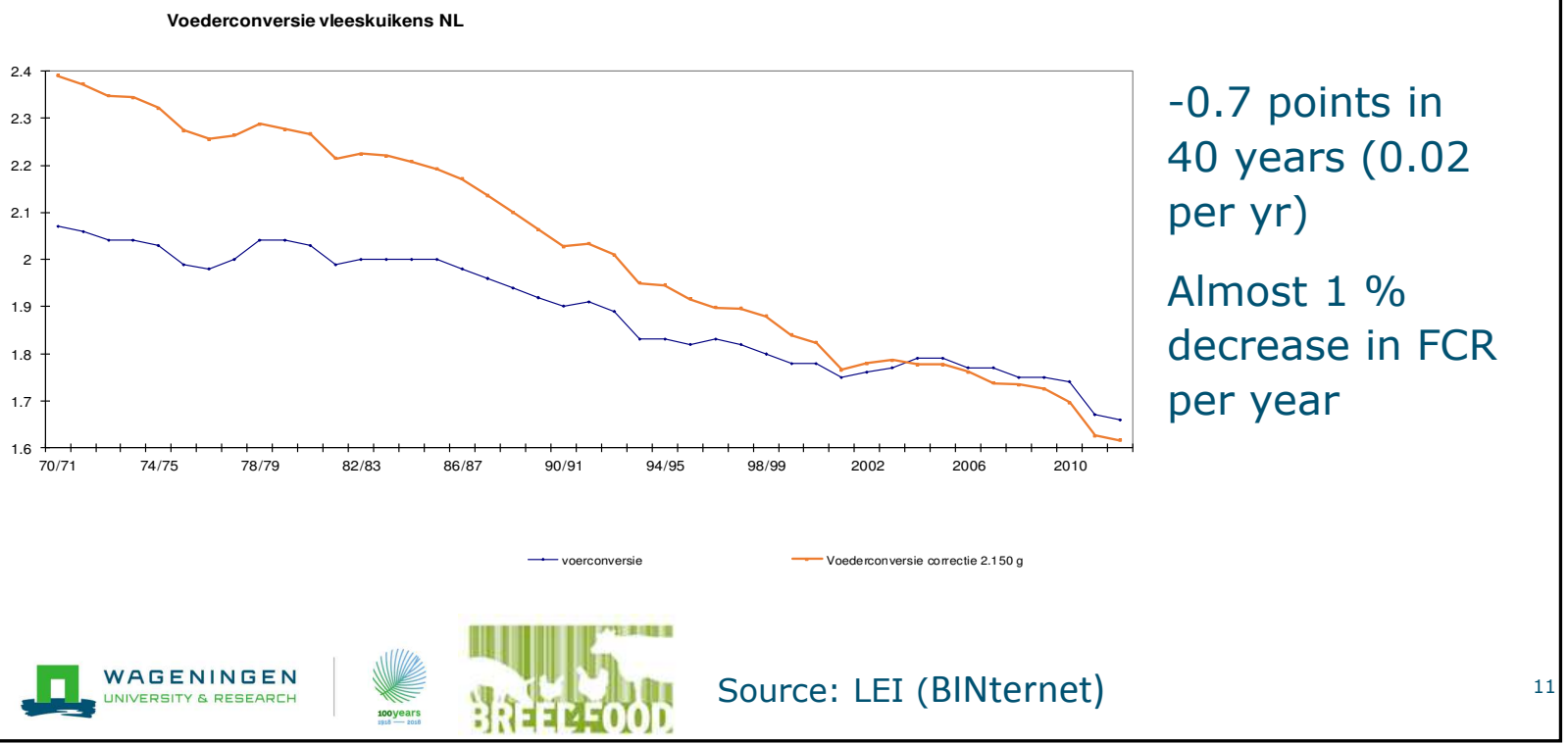




\section{Broilers - Limits to ongoing success?}

Conclusions by

Tallentire et al., based on modelling approach:

- Growth rate close to physiological maximum

- Alternative breeding strategy to slowgrowing (56 days) will increase resource use

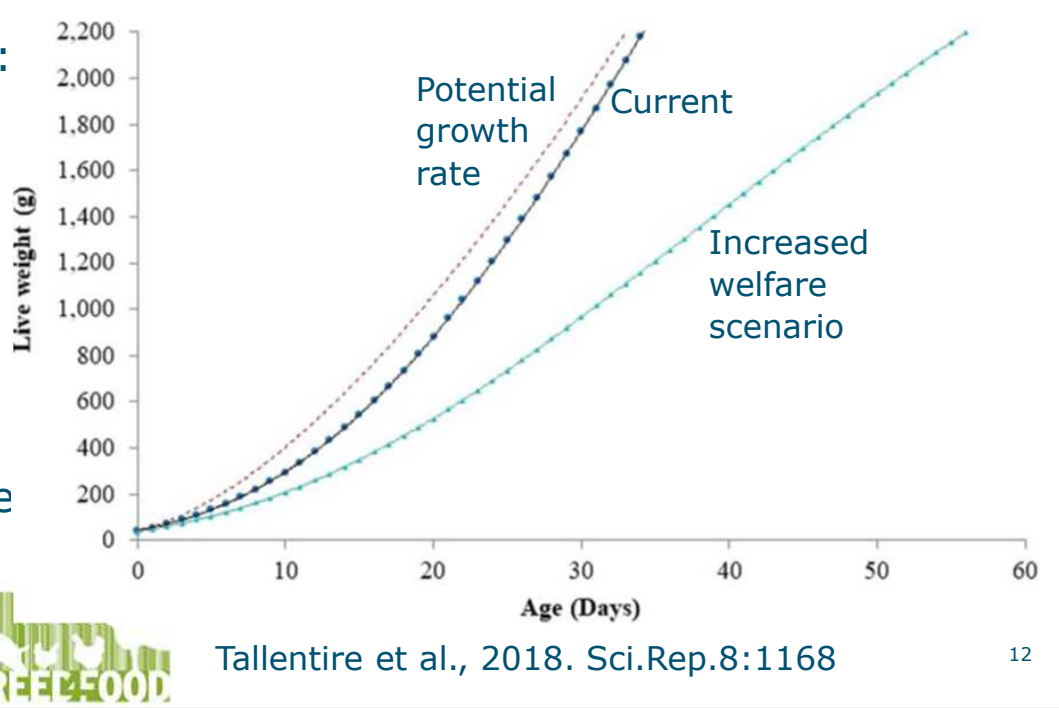




\subsection{Historical trends - Layers}

\section{Layers - GHG emissions}

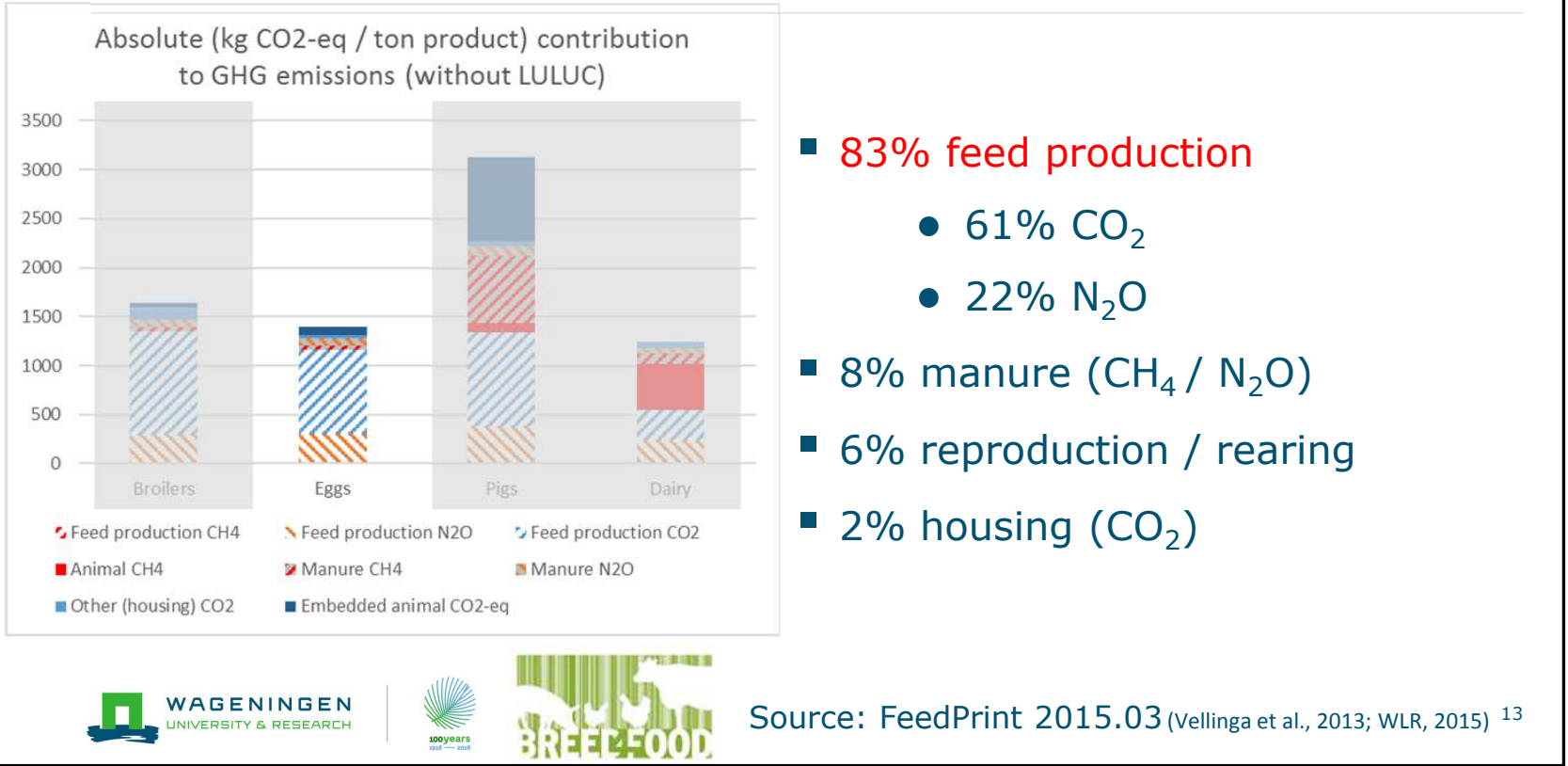

\section{Layers - LCA historical / international1,2}

- Canada 1962 - 2012: GHG emissions reduced by $72 \%$

- US 1960 - 2010: GHG emissions reduced by 71\%

- 3 primary factors:

- feed efficiency

- feed composition

- manure management 


\section{Layers - feed conversion and production}

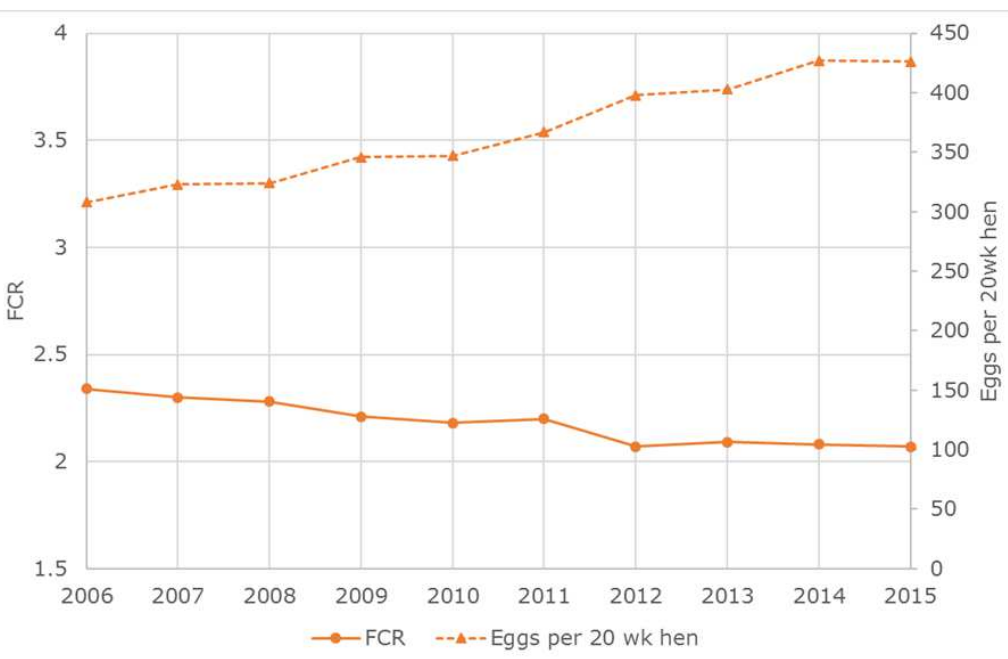

\# eggs: Increase, mainly due to longer production period

CR: Slight decrease, but seems to flatten

\section{Layers - LCA methodology (MSc thesis)}

- Assessment of progress based on:

- Assumed goals for annual genetic improvement

$$
\text { Trait }
$$

Brown lines

White lines

Total production

Survival rearing

+2.3 eggs

+2.5 eggs

Survival production

Average egg weight

Feed intake

$+0.05 \%$

$+0.15 \%$

$+0.1 \mathrm{~g}$

0

$+0.1 \mathrm{~g}$

0

- Sensitivity analysis based on genetic standard deviations

\section{Trait}

Survival rearing (\%)

Survival production (\%)

Egg weight (g)

Egg production (number of eggs) $3.82^{2}$

- Assumed goal (500 eggs in 100 weeks) 


\section{Layers - LCA results (MSc thesis)}

- Annual genetic improvement: $0.87-0.92 \%$ reduction in GHG

- Sensitivity analysis (per 1 gen.std.dev)

- Most sensitive for egg production or egg weight (both $3.8 \%$ )

- 500 eggs in 100 weeks compared to 360 eggs in 80 weeks

- $6 \%$ reduction in GHG, mainly due to improvement of FCR 


\section{$2.4 \quad$ Historical trends - Pigs}

\section{Pigs - GHG emissions}

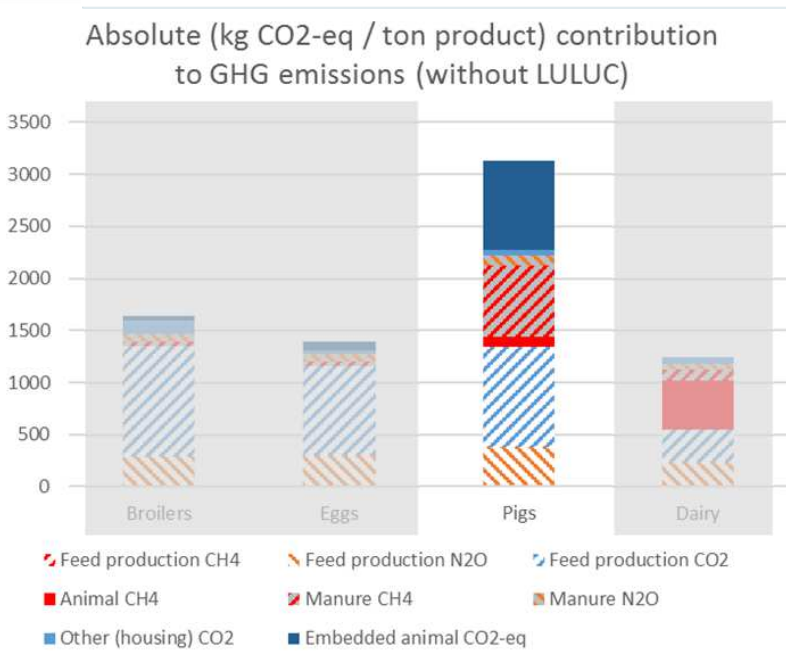

- $43 \%$ feed production

- $31 \% \mathrm{CO}_{2}$

- $12 \% \mathrm{~N}_{2} \mathrm{O}$

- $27 \%$ reproduction / rearing

- $25 \%$ manure

- $22 \% \mathrm{CH}_{4}$

- $3 \% \mathrm{~N}_{2} \mathrm{O}$

- $3 \%$ animal $\mathrm{CH}_{4}$

- $2 \%$ housing $\left(\mathrm{CO}_{2}\right)$

\section{Pigs - FCR growing-finishing pigs (1982 - 2012)}

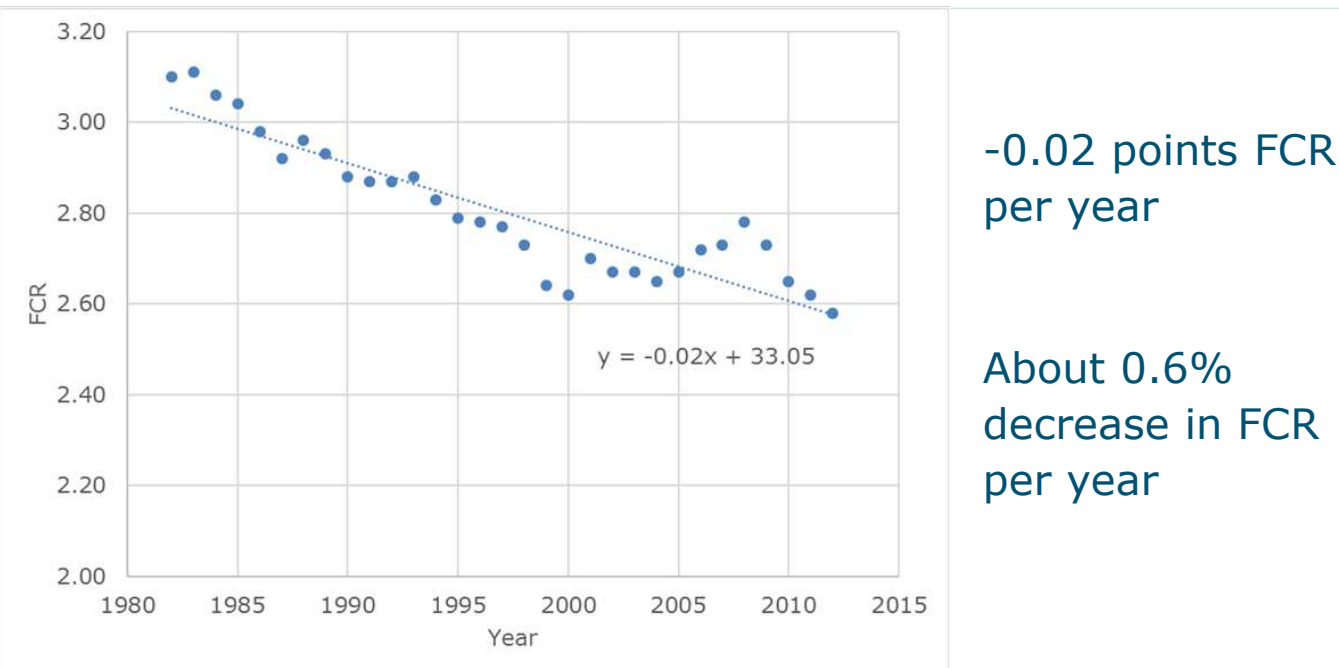




\section{Pigs - reproduction sows (1986 - 2013)}

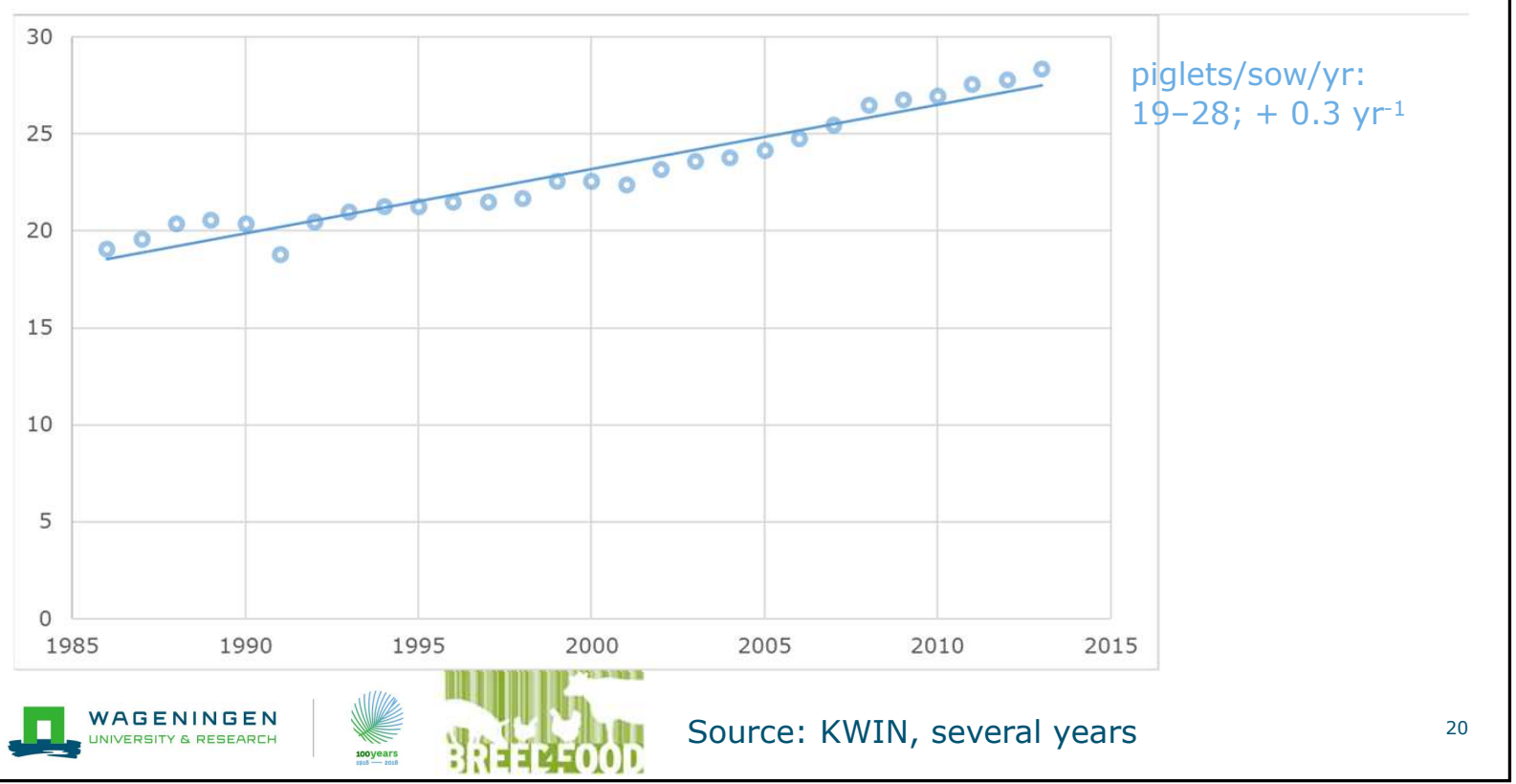

\section{Pigs - sensitivity analysis ${ }^{1}$}

- Search for most important factor in GHG model

- LCA - whole production chain

- Sensitivity analysis - unique and sophisticated method

- Conclusion: FCR most important factor

- Reproduction performance - rather sensitive, but variation in practice is relatively low 


\subsection{Historical trends - Dairy}

\section{Dairy - GHG emissions}

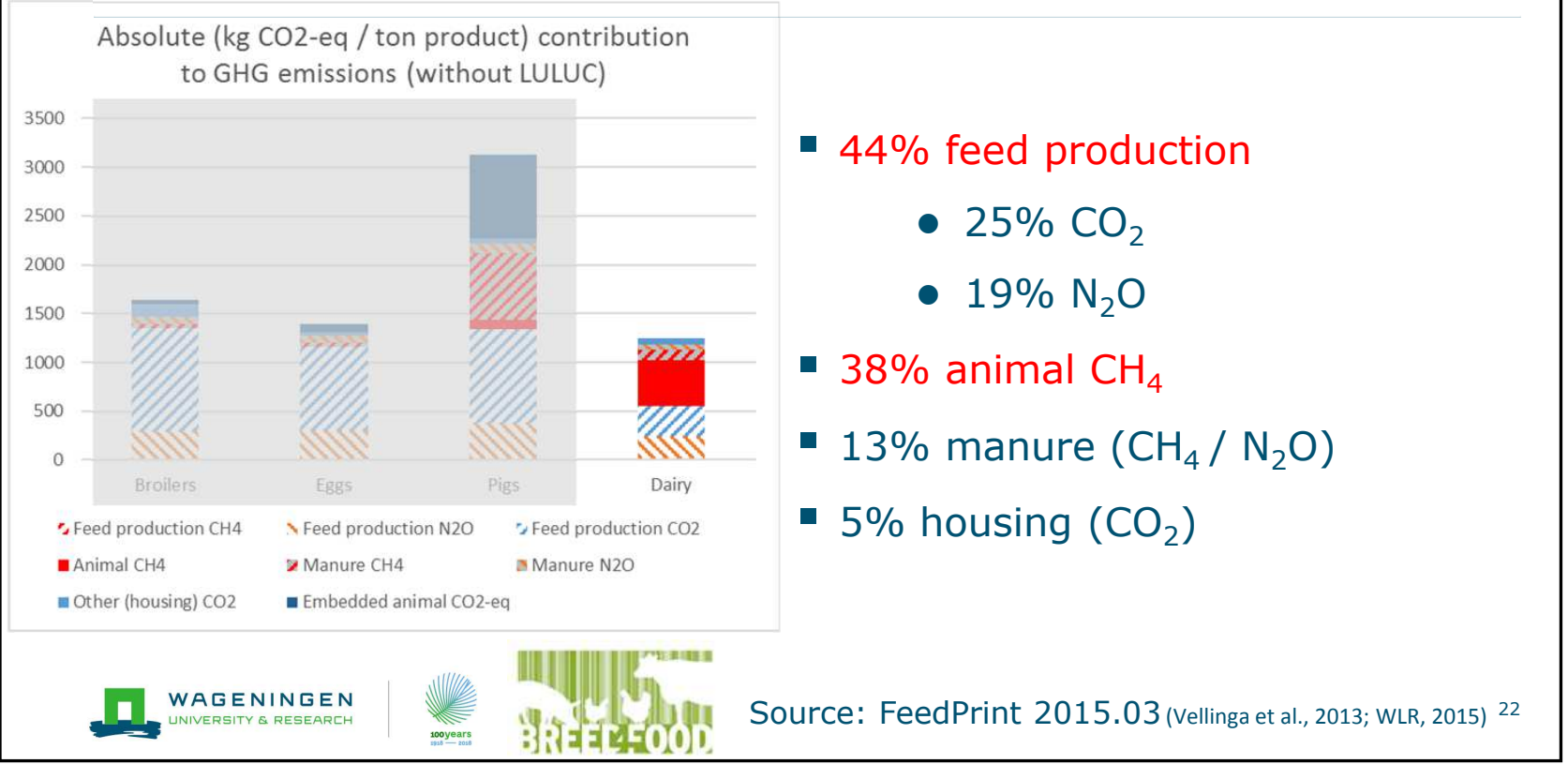

\section{Dairy - production efficiency}

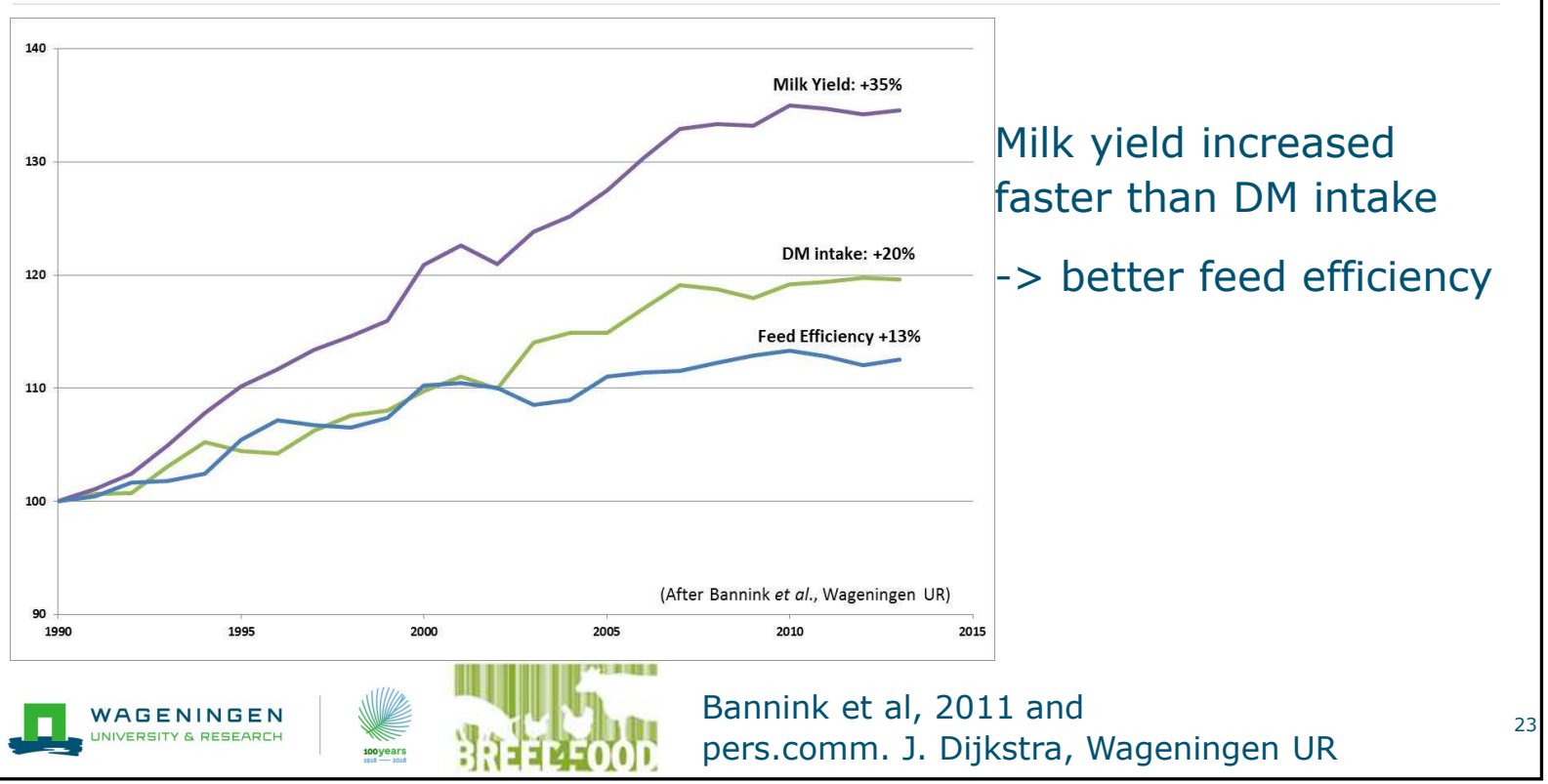




\section{Dairy - enteric $\mathrm{CH}_{4}$ emission}

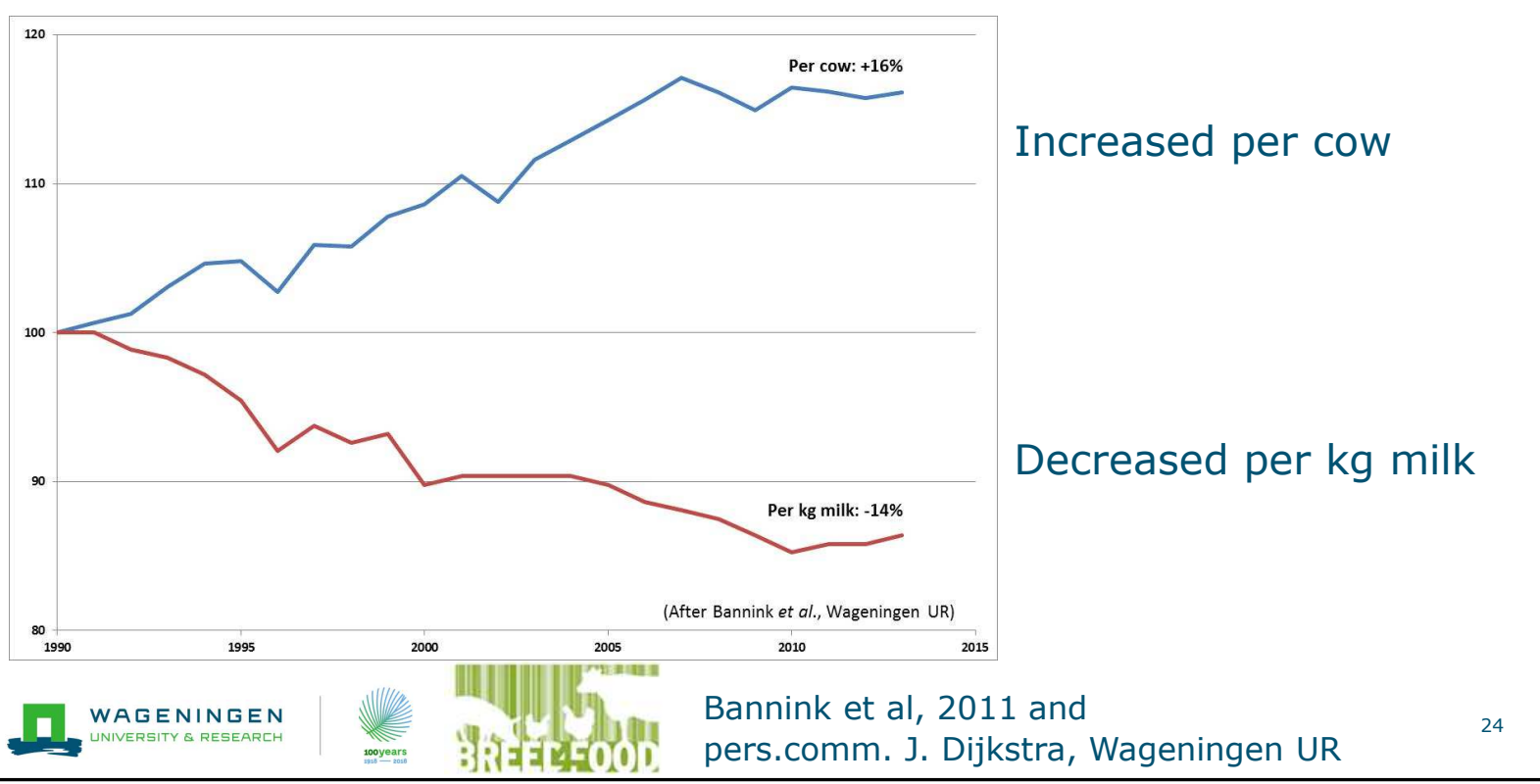

\section{Dairy - reduction of $\mathrm{GHG}^{1}$}

Carbon footprint dairy

$\left.\begin{array}{ll}\text { 1990: } 2.06 \mathrm{~kg} \mathrm{CO} \text {-eq. / kg milk } \\ \text { 2012: } 1.42 \mathrm{~kg} \mathrm{CO} \text {-eq. / kg milk }\end{array}\right\}-31 \%$ 


\subsection{Conclusions and Discussion literature review}

\section{Conclusions from literature review}

The contribution of breeding to reducing environmental impact of animal production

- Indirect response through increasing efficiency

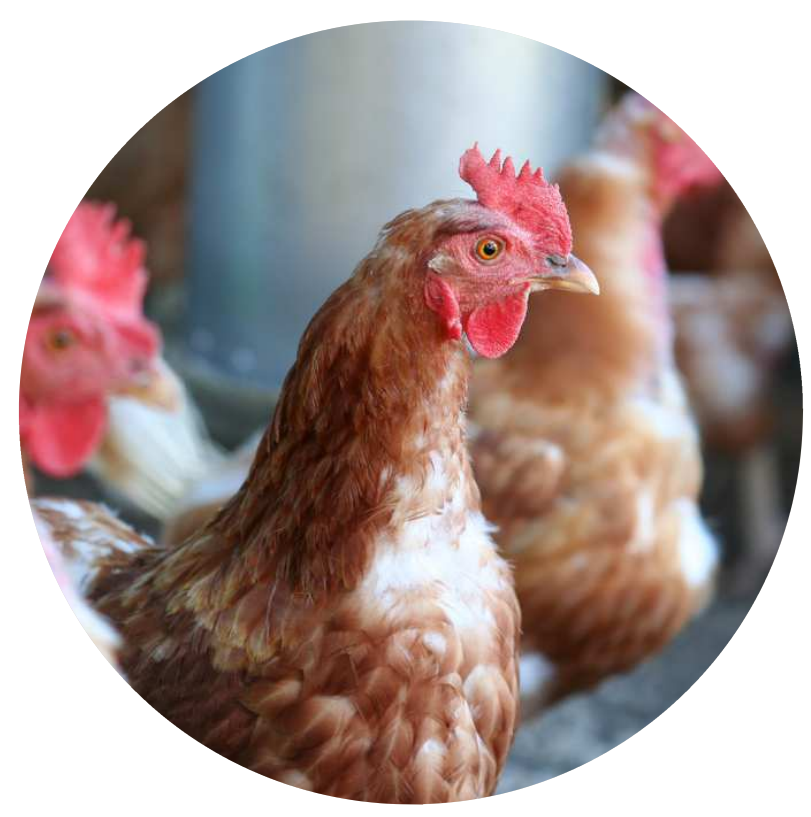

\section{Discussion with B4F partners}

- In general (pigs and poultry) no specific focus on GHG reduction

- Main 'source' feed production, covered by feed efficiency

- Very limited contribution of direct animal related emissions

- More interested in N and P efficiency (animal level)

- Exception, dairy, where enteric $\mathrm{CH}_{4}$ emission is large contributor to GHG emissions 


\section{Layers - discussion}

- Strong relation feed efficiency - env. impact

- Most important in breeding goal: more eggs

- Little/no information available on (variation in) N/P efficiency

- "What is the future feed composition?"

- Literature (based on report Ellen et al - protein efficiency)

- Differences in how lines deal with different protein sources

- Possibilities to change from soybean meal to other protein source 


\section{Quantification of contribution of animal breeding}

\section{Quantification - overview}

Broilers, layers, and pigs

- GHG emissions and $\mathrm{N}$ and $\mathrm{P}$ efficiency

- Where possible, cradle to farm gate LCA, otherwise, as indicated, part of production chain

- GHG impact and P and crude protein content of feed components from FeedPrint 2018 (Vellinga et al., 2013; WLR, 2018)

Dairy

- Effect of genetic progress through correlated responses 


\subsection{Quantification - Broilers}

\section{Broilers - information from Cobb}

- Genetic progress, current feed

- Data commercial flocks

\begin{tabular}{|lccccccc|}
\hline Year & First & Last & \# flocks & $\begin{array}{c}\text { Avg. } \\
\text { numb. }\end{array}$ & $\begin{array}{c}\text { Avg. } \\
\text { age }\end{array}$ & $\begin{array}{c}\text { Avg. } \\
\text { weight }\end{array}$ & $\begin{array}{c}\text { Avg. } \\
\text { FCR }\end{array}$ \\
\hline 2014 & $07-05-13$ & $28-08-14$ & 12 & 33105 & 37.2 & 2.2 & 1.66 \\
\hline 2018 & $16-06-17$ & $07-08-18$ & 10 & 63716 & 40.3 & 2.7 & 1.56 \\
\hline
\end{tabular}

- Linear extrapolation of genetic progress

\section{Broilers - quantification - methods}

- Broiler production phase only

- Variables derived from dataset:

- Feed Conversion Ratio (FCR)

- Final body weight

- Feed composition from FeedPrint 2015.03 (Vellinga et al., 2013; WLR, 2015)

- $10 \%$ starting / 90\% growing feed

- $\mathrm{N}$ and $\mathrm{P}$ in whole animal after 1 day fasting based on Caldas (2015) 


\section{Broilers - GHG results genetic progress}

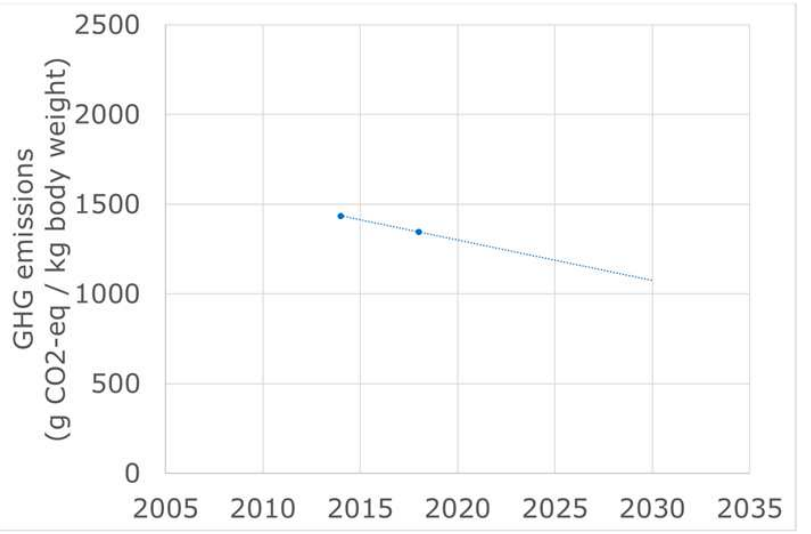

Genetic progress

-23 $\mathrm{g} \mathrm{CO}_{2}$-eq per $\mathrm{kg}^{\mathrm{a}}$ per $\mathrm{yr}$

$-1.7 \%$ per $y r$

Predicted performance (2030)

-270 $\mathrm{g} \mathrm{CO}_{2}$-eq per kg body weight $^{\mathrm{a}}(20.1 \%)$ compared to current

\section{Broilers - P efficiency results genetic progress}

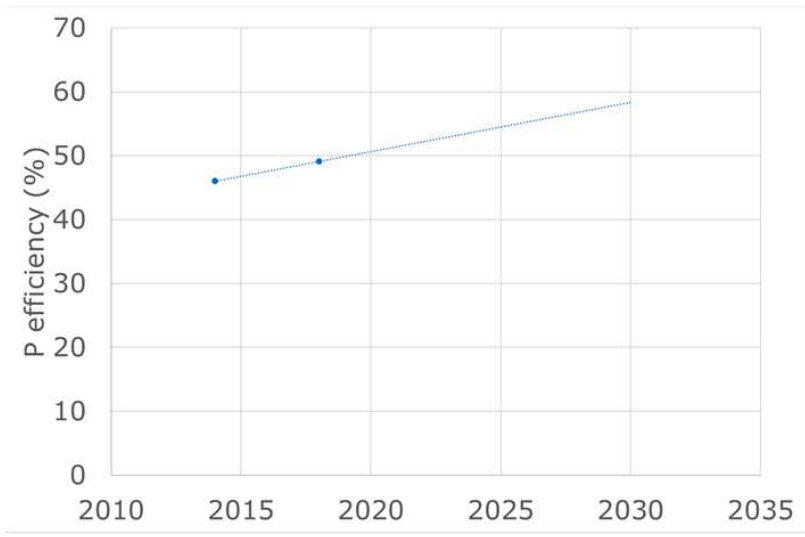

Genetic progress

$+0.77 \%$-points per $\mathrm{yr}$

$+1.6 \%$ per yr

Predicted performance (2030)

$+9.2 \%$ points $(18.8 \%)$ compared to current 


\section{Broilers - $\mathrm{N}$ efficiency results genetic progress}

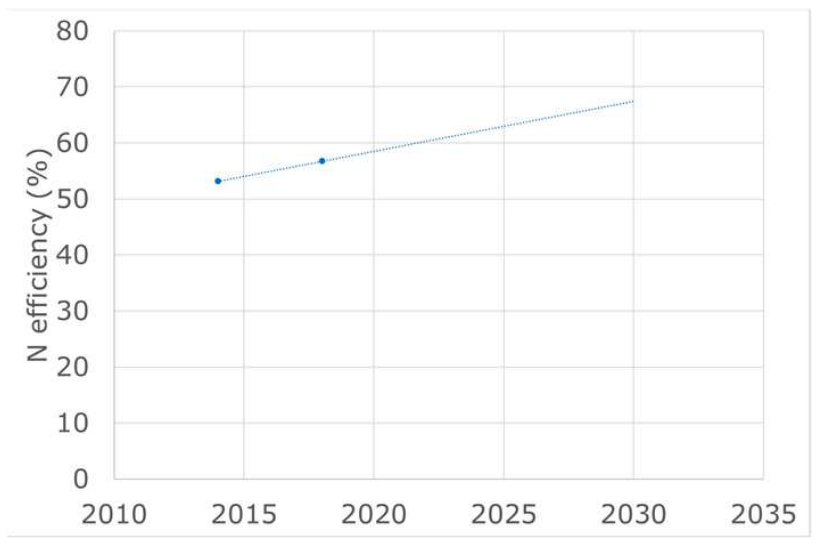

Genetic progress

$+0.89 \%$-points per yr

$+1.6 \%$ per yr

Predicted performance (2030)

$+10.7 \%$ points

(18.8\%) compared to

current

\section{Broilers - discussion}

- Decrease in FCR in dataset (1.5\% per yr) stronger than expected from historic overview from literature (1\% per yr)

- Increase in flock size (almost doubled in 4 yrs) in dataset

- Increase in age at slaughter ( +3 days in 4 yrs) in dataset and, therefore, increase in final body weight ( 2.2 to $2.7 \mathrm{~kg}$ )

- May cause overestimation of genetic progress 


\section{Broilers - discussion (2)}

- Only accounted for feed intake broiler production phase

- $\mathrm{N}$ and $\mathrm{P}$ efficiency based on whole body including non-edible parts

- $\mathrm{N}$ and $\mathrm{P}$ in final product (edible parts) needs to be better known to calculate $\mathrm{N}$ and $\mathrm{P}$ efficiency more accurately

\section{Broilers - conclusions}

- GHG emissions decrease and $\mathrm{N}$ and $\mathrm{P}$ efficiency increase with current breeding goal 


\subsection{Quantification - Layers}

\section{Layers - information from Hendrix Genetics}

- Current situation, current feed

- Brown (80 wks) and white (90 wks) lines

- Based on product guides for alternative systems

- Genetic progress, current feed

- Brown and white lines

- Based on top performers

- See next slides: "Development of the modern Brown/White commercial layer" comparing 2008/2009 (75 wks) vs 2017 (90 wks)

\section{Development of the modern Brown commercial laver}

\begin{tabular}{l}
\hline HH EGGS AT 75 Weeks \\
\hline HH EGGS AT 90 Weeks \\
\hline HH EGGS AT 100 Weeks \\
\hline
\end{tabular}

\begin{tabular}{|c|c|c|c|c|c|}
\hline (NRS) & 239 & 306 & 324 & 350 & 361 \\
\hline$(N R S)$ & & & & 429 & 446 \\
\hline$(N R S)$ & & & & & 500 \\
\hline$(W K S)$ & 26 & 20 & 20 & 20 & 20 \\
\hline$(W K S)$ & 29 & 26 & 26 & 25 & 25 \\
\hline$(\%)$ & 86 & 95 & 96 & 97 & 97 \\
\hline$(K G)$ & 14.9 & 19.2 & 20.6 & 21.9 & 22.6 \\
\hline$(K G)$ & & & & 27.0 & 28.0 \\
\hline$(K G)$ & & & & & 31.5 \\
\hline$(G / D)$ & 127 & 114 & 114 & 113 & 112 \\
\hline$(K G / K)$ & 3.46 & 2.41 & 2.25 & 2.14 & 2.07 \\
\hline$(\%)$ & 90 & 94 & 94 & 95 & 95 \\
\hline$(\%)$ & 55 & 74 & 76 & 80 & 82 \\
\hline$(K G S)$ & 1.72 & 1.55 & 1.55 & 1.50 & 1.50 \\
\hline$(K G S)$ & 2.5 & 2.0 & 2.0 & 2.0 & 1.9 \\
\hline
\end{tabular}




\section{Development of the modern White commercial layer}

\begin{tabular}{|c|c|c|c|c|c|c|}
\hline & & 1970 & 2004 & 2009 & 2017 & 2020 \\
\hline HH EGGS AT 75 Weeks & (NRS) & 250 & 315 & 329 & 353 & 364 \\
\hline HH EGGS AT 90 Weeks & (NRS) & & & & 433 & 449 \\
\hline HH EGGS AT 100 Weeks & (NRS) & & & & & 505 \\
\hline AGE AT 50\% PRODUCTION & (WKS) & 24 & 20 & 20 & 20 & 20 \\
\hline AGE AT PEAK PRODUCTION & (WKS) & 27 & 26 & 25 & 25 & 25 \\
\hline RATE OF LAY AT PEAK & $(\%)$ & 88 & 95 & 96 & 97 & 97 \\
\hline EGG MASS AT 75 Weeks & $(K G)$ & 15,4 & 19,6 & 20,7 & 22,0 & 22,7 \\
\hline EGG MASS AT 90 Weeks & $(K G)$ & & & & 27,3 & 28,3 \\
\hline EGG MASS AT 100 Weeks & $(K G)$ & & & & & 32,0 \\
\hline FEED/DAY & $(G / D)$ & 115 & 110 & 110 & 109 & 109 \\
\hline FCR resp. 75 to 90 to 100 weeks of age & $(K G / K)$ & 3,03 & 2,28 & 2,16 & 2,05 & 1,98 \\
\hline LIVEABILITY & $(\%)$ & 90 & 94 & 94 & 95 & 95 \\
\hline HEN DAY RATE OF LAY AT 75 Weeks & $(\%)$ & 60 & 75 & 76 & 82 & 84 \\
\hline BODYWEIGHT AT 18 Weeks & (KGS) & 1,4 & 1,3 & 1,3 & 1,3 & 1,3 \\
\hline ADULT BODYWEIGHT & (KGS) & 1,8 & 1,7 & 1,7 & 1,7 & 1,7 \\
\hline
\end{tabular}

\section{Layers - quantification - methods}

- GHG based on whole chain incl. parent stock and rearing ${ }^{1}$

- Feed composition from FeedPrint 2015.03 (Vellinga et al., 2013; WLR, 2015)

- $\mathrm{N}$ and $\mathrm{P}$ efficiency based on laying period only ${ }^{2}$

- Linear extrapolation of genetic progress

- Application of percentage wise increase on current performance to predict performance in 2030

\footnotetext{
${ }^{1}$ For calculation genetic progress only laying performance adapted
} 


\section{Layers - GHG results current situation}

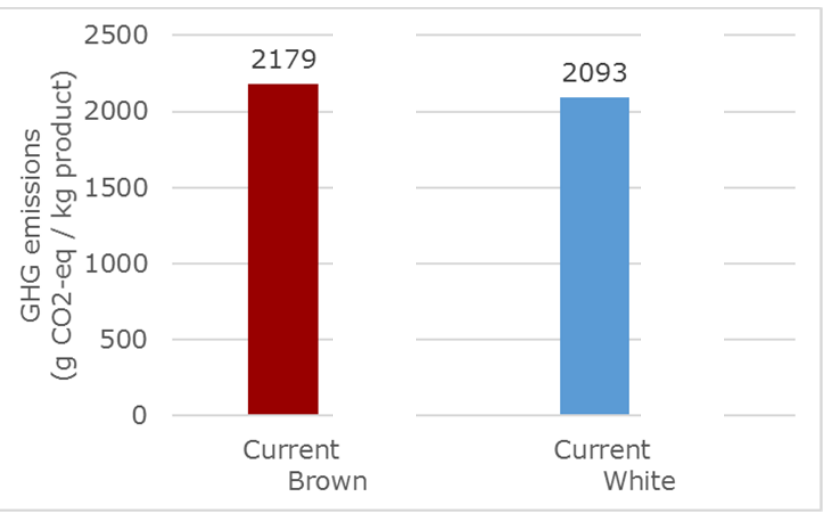

Current performance (2018)

for alternative systems

Brown $87 \mathrm{~g} \mathrm{CO}_{2}$-eq per $\mathrm{kg}$ egga higher $(4.1 \%)$ compared to white

\section{Layers - GHG results genetic progress}

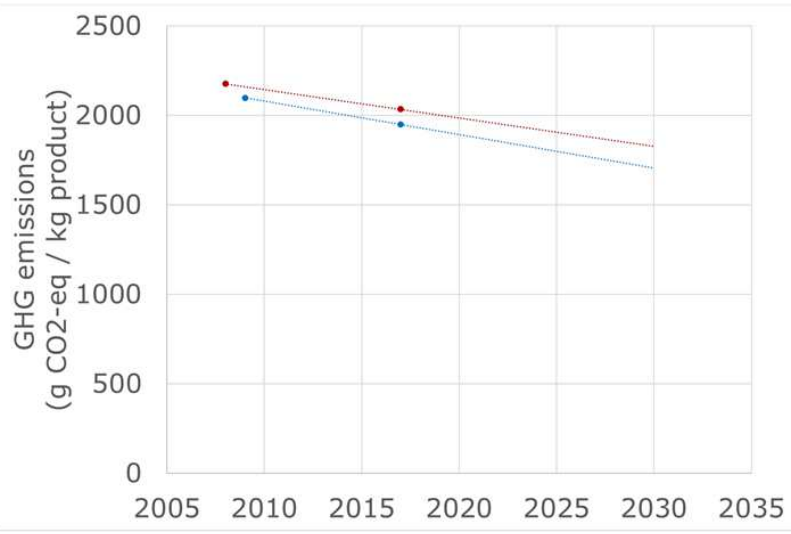

Brown

-16 $\mathrm{g} \mathrm{CO}_{2}$-eq per $\mathrm{kg}^{\mathrm{a}}$ per $\mathrm{yr}$

$-0.8 \%$ per yr

White

-19 $\mathrm{g} \mathrm{CO}_{2}$-eq per $\mathrm{kg}^{\mathrm{a}}$ per $\mathrm{yr}$

$-1.0 \%$ per yr 


\section{Layers - GHG results prediction}

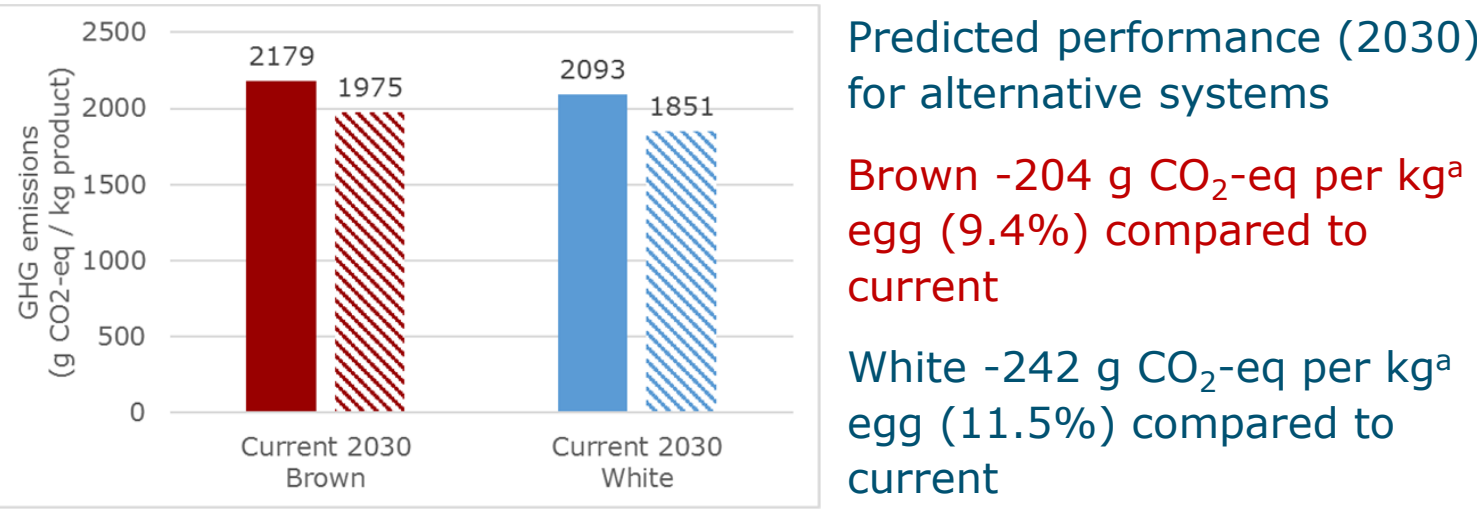

\section{Layers - P efficiency results current situation}

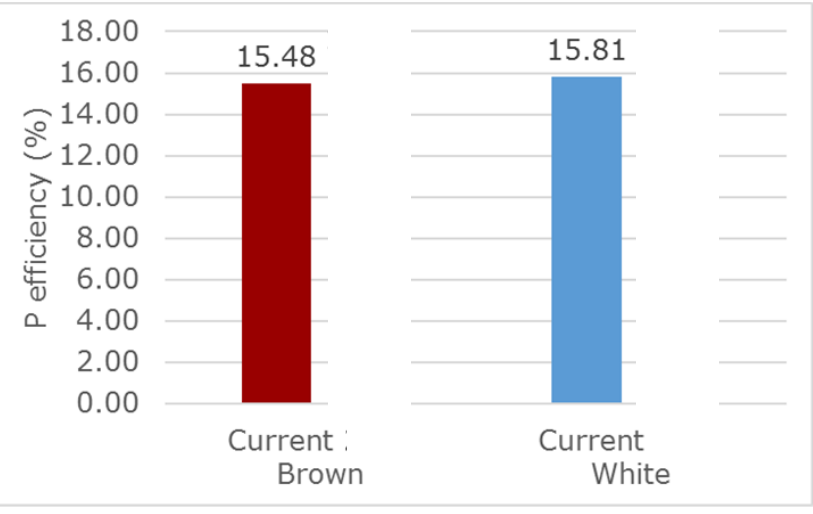

Current performance (2018) for alternative systems

Brown $0.33 \%$-points lower $(2.1 \%)$ compared to white 


\section{Layers - P efficiency results genetic progress}

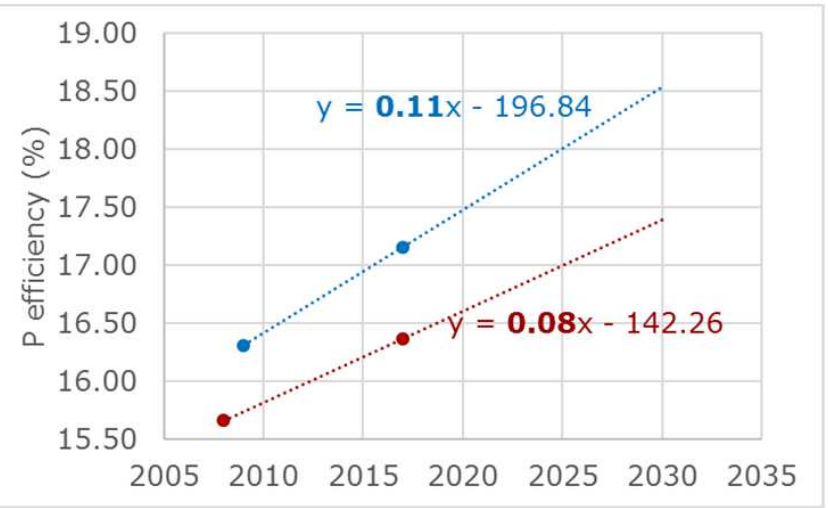

White

$$
\begin{aligned}
& +0.11 \% \text {-points per yr } \\
& +0.62 \% \text { per } y r
\end{aligned}
$$

Brown

$$
\begin{aligned}
& +0.08 \% \text {-points per yr } \\
& +0.48 \% \text { per } y r
\end{aligned}
$$

\section{Layers - P efficiency results prediction}

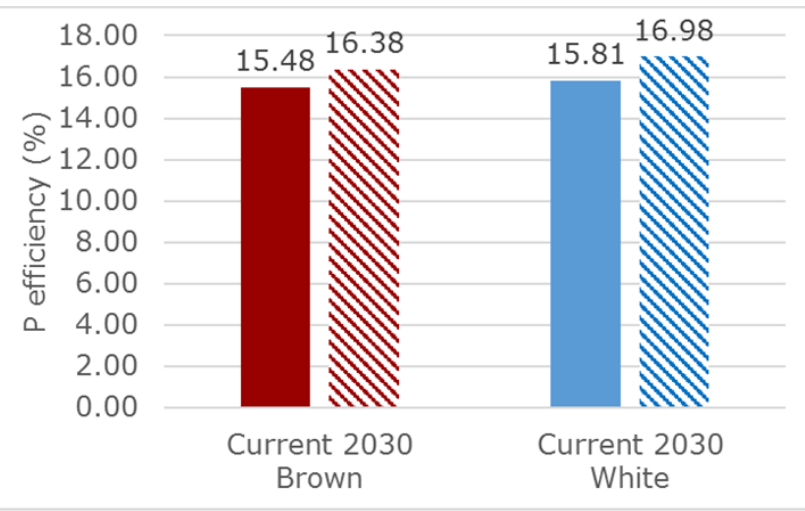

Predicted performance (2030) for alternative systems

Brown $+0.89 \%$-points (5.8\%) compared to current

White $+1.17 \%$-points (7.4\%) compared to current 


\section{Layers - $\mathrm{N}$ efficiency results prediction}

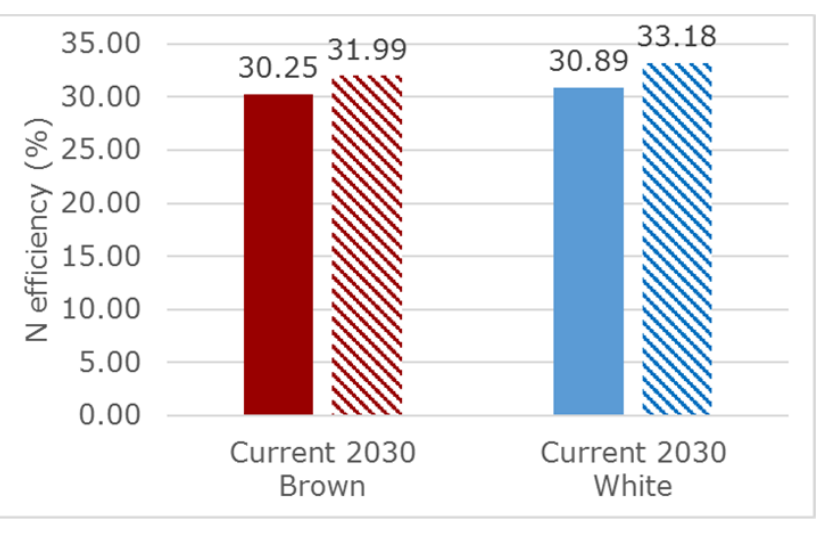

Current performance (2018)

Brown $0.64 \%$-points lower

$(2.1 \%)$ compared to white

Predicted performance (2030)

Brown $+1.74 \%$-points

(5.8\%) compared to current

White $+2.29 \%$-points $(7.4 \%)$

compared to current

\section{Layers - discussion}

- GHG emissions based on whole chain LCA, but improvement only accounted for egg production phase

- $\mathrm{N}$ and $\mathrm{P}$ efficiency based on edible part of egg and feed input in egg production phase

- Risk of breeding for thin egg shells?

- $\mathrm{N}$ and $\mathrm{P}$ in final product (eggs) needs to be better known to calculate $\mathrm{N}$ and $\mathrm{P}$ efficiency more accurately 


\section{Layers - conclusions}

- GHG emissions decrease and $\mathrm{N}$ and $\mathrm{P}$ efficiency increase with current breeding goal

- White hens perform already better, and improve faster than brown hens 


\subsection{Quantification - Pigs}

\section{Pigs - information from Topigs-Norsvin}

- Genetic progress, 2 types of feed

- Data from experiment ${ }^{1}$

- Corn / soy (CS) $)^{2}$ diet vs cereals / alternative ingredients (by-products) (CA) diet

- Male (intact boars) vs female (gilts)

- 400 pigs in 2014 (Dec'13-May'14)

- 401 pigs in 2016 (Nov'15-Mar'16)

- Linear extrapolation of genetic progress

\section{Pigs - quantification - methods}

- Growing-finishing phase only (from about $22 \mathrm{~kg}$ onwards)

- Feed composition (Sevillano et al., 2018)

- Variables derived from experiment:

- Feed intake (starter/grower/finisher)

- Body weight gain

- Empty body weight (EBW) at slaughter

- Protein deposition

- P deposition in EBW based on Pettey et al. (2015) 


\section{Pigs - GHG results genetic progress}

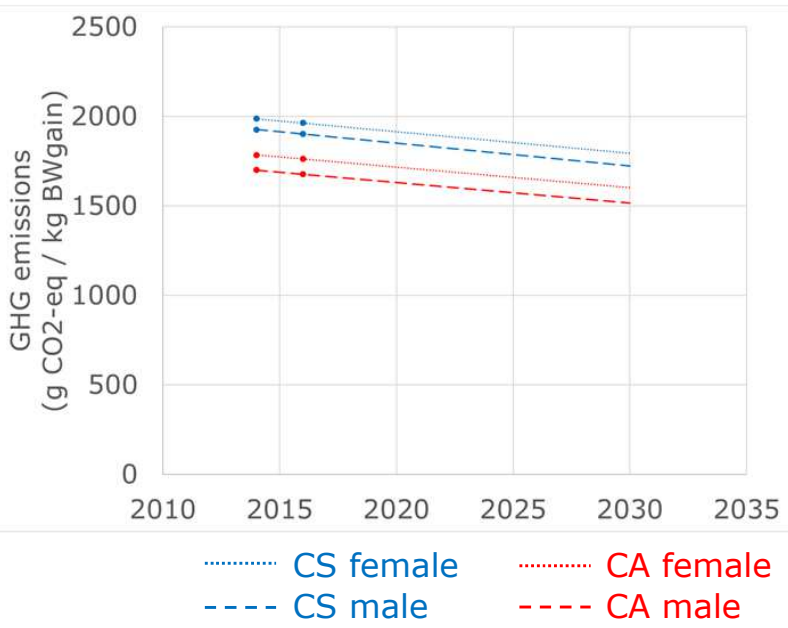

Corn / soy diet

q 3\% higher than $\widehat{\jmath}$

- $12 \mathrm{~g} \mathrm{CO}_{2}$-eq per kga per yr

$-0.6 \%$ per yr

Cereals / alternative diet

ㅇ $5 \%$ higher than $\hat{\sigma}$

-12 $\mathrm{g} \mathrm{CO}_{2}$-eq per $\mathrm{kg}^{\mathrm{a}}$ per yr

$-0.7 \%$ per yr

\section{Pigs - P efficiency results genetic progress}

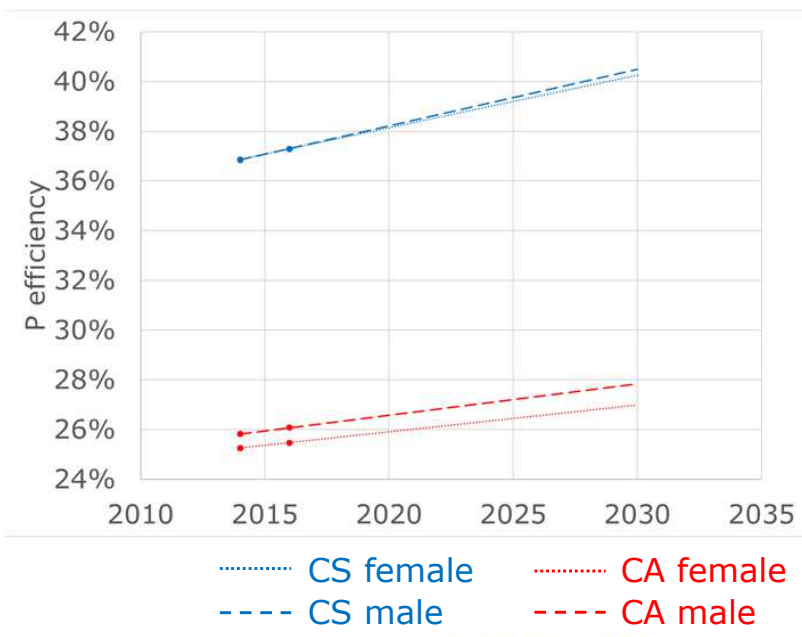

Corn / soy diet

q equal to $\delta$

$+0.22 \%$-points per $y r$

$+0.6 \%$ per yr

Cereals / alternative diet

q $2 \%$ lower than $\delta^{\lambda}$

$+0.12 \%$-points per yr

$+0.5 \%$ per yr 


\section{Pigs - N efficiency results genetic progress}

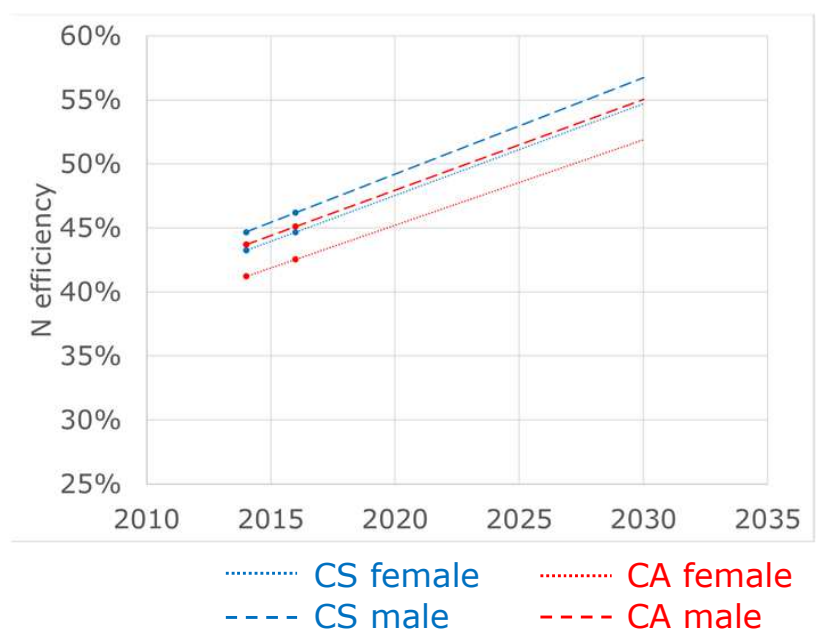

WAGENINGEN
Corn / soy diet

+ $3 \%$ lower than ${ }^{\lambda}$

$+0.73 \%$-points per $\mathrm{yr}$

$+1.6 \%$ per yr

Cereals / alternative diet

q $6 \%$ lower than $\delta$

$+0.69 \%$-points per $\mathrm{yr}$

$+1.6 \%$ per yr

\section{Pigs - discussion}

- Only accounted for feed intake in growing-finishing phase

- Corn-soy diet as fed in Europe; impact when produced and fed in same country expected to be lower

- Low P efficiency on CA diet due to low digestibility of P in some byproducts (e.g. rapeseed and sunflower meal) 


\section{Pigs - conclusions}

- GHG emissions decrease and $\mathrm{N}$ and $\mathrm{P}$ efficiency increase with current breeding goal

- Boars perform slightly better than gilts 


\subsection{Quantification - Dairy}

\section{Dairy - quantification}

- Correlated responses to mimic the effect of

- Methane added to current breeding goal

- With or without data on methane emissions of individual cows

- With what economic weight?

\section{Standard cow}

\section{5d milking}

$60 d$ dry

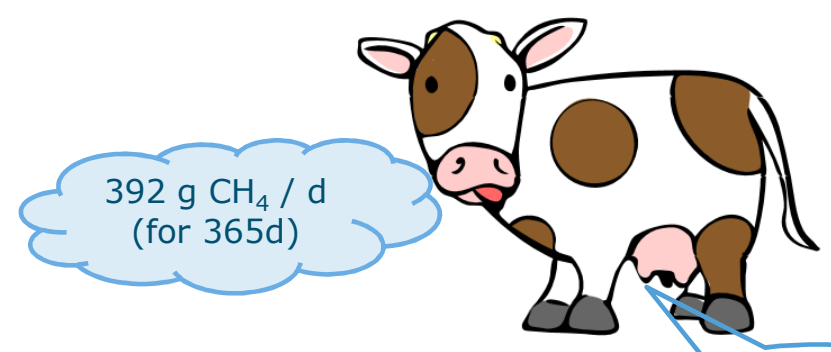

$9000 \mathrm{~kg}$ milk / $305 \mathrm{~d}$ 


\section{Genetic parameters methane production $(\mathrm{g} / \mathrm{d})$}

Methane production (Lassen en Lovendahl, 2016)

- Phen std: $36 \mathrm{~g} / \mathrm{d}$

- $\mathrm{h}^{2}: 0.21$

- Genetic correlations:

\begin{tabular}{|llll|} 
Lactose & Fat & Protein & $\begin{array}{l}\text { Saved feed } \\
\text { costs }\end{array}$ \\
\hline $0.43^{1}$ & $0.37^{2}$ & $0.77^{2}$ & $-0.42^{3}$ \\
\hline
\end{tabular}

- All other correlations are set to 0

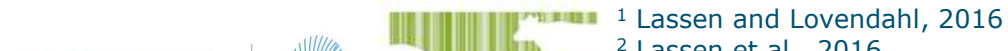

\section{Value for methane production}

Methane

- Expected carbon price in $2025^{1}: 36.19 €$ per tonne

- Low = 10€; high = $100 €$

- Global warming potential: $28 \mathrm{~g} \mathrm{CO}_{2}$-eq / g CH

- Economic value: $-0.37 € /$ year

$$
-1 *(36.19 * 28 / 1,000,000) * 365
$$

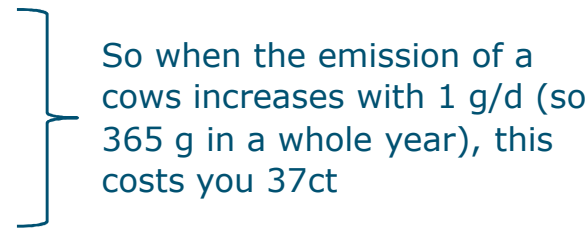




\section{Genetic gain methane (g/d) \& Economic value}

\begin{tabular}{|lcccccc} 
& $\begin{array}{l}\text { NVI w } \\
\mathbf{C H}_{\mathbf{4}}\end{array}$ & $\begin{array}{l}\text { No gain } \\
\mathbf{C H}_{\mathbf{4}}\end{array}$ & $\begin{array}{l}\text { Regular } \\
\text { econ. } \\
\text { value }\end{array}$ & $\begin{array}{l}\text { Low } \\
\text { econ. } \\
\text { value }\end{array}$ & $\begin{array}{l}\text { High } \\
\text { econ. } \\
\text { value }\end{array}$ \\
\hline Gain & 5.77 & 0.00 & 4.93 & 5.54 & 3.31 \\
\hline Econ value & 0.00 & -2.24 & -0.37 & -0.10 & -1.02 \\
\hline
\end{tabular}

\section{Methane production and intensity per cow}

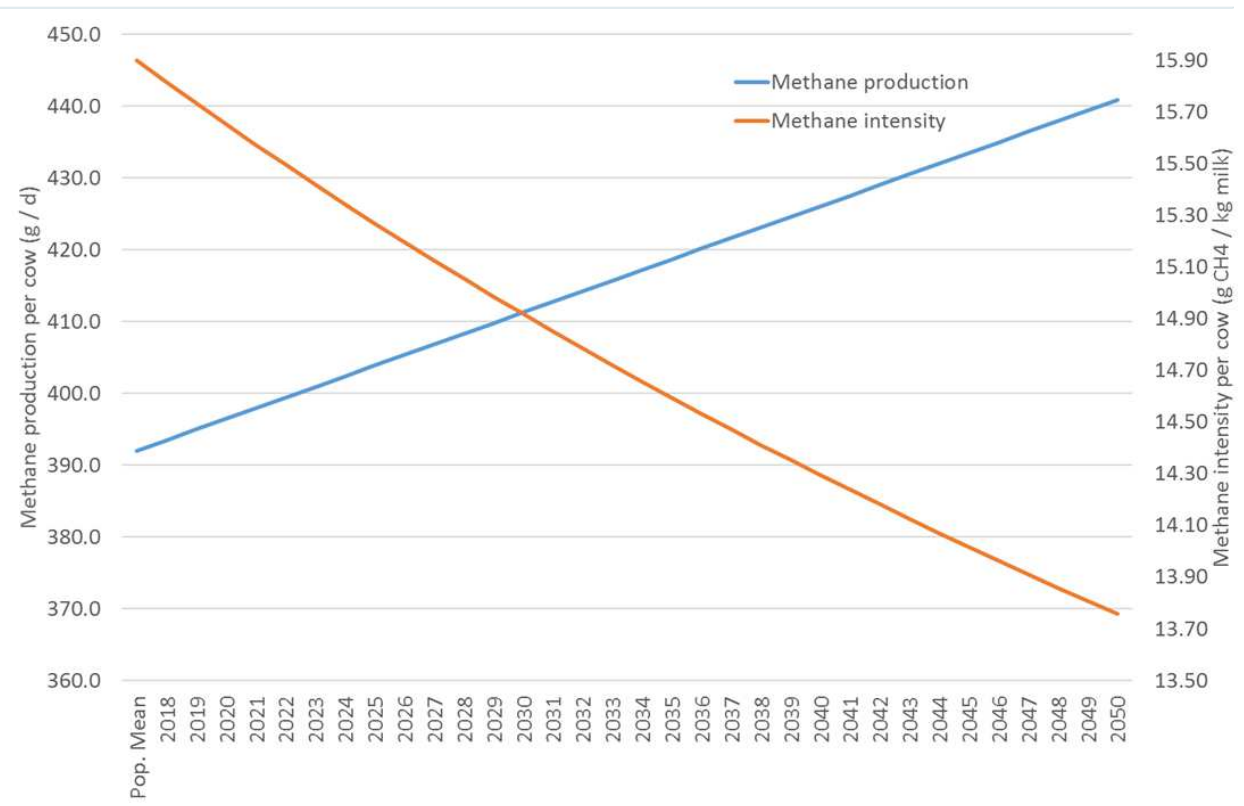




\section{Methane production and intensity per cow}

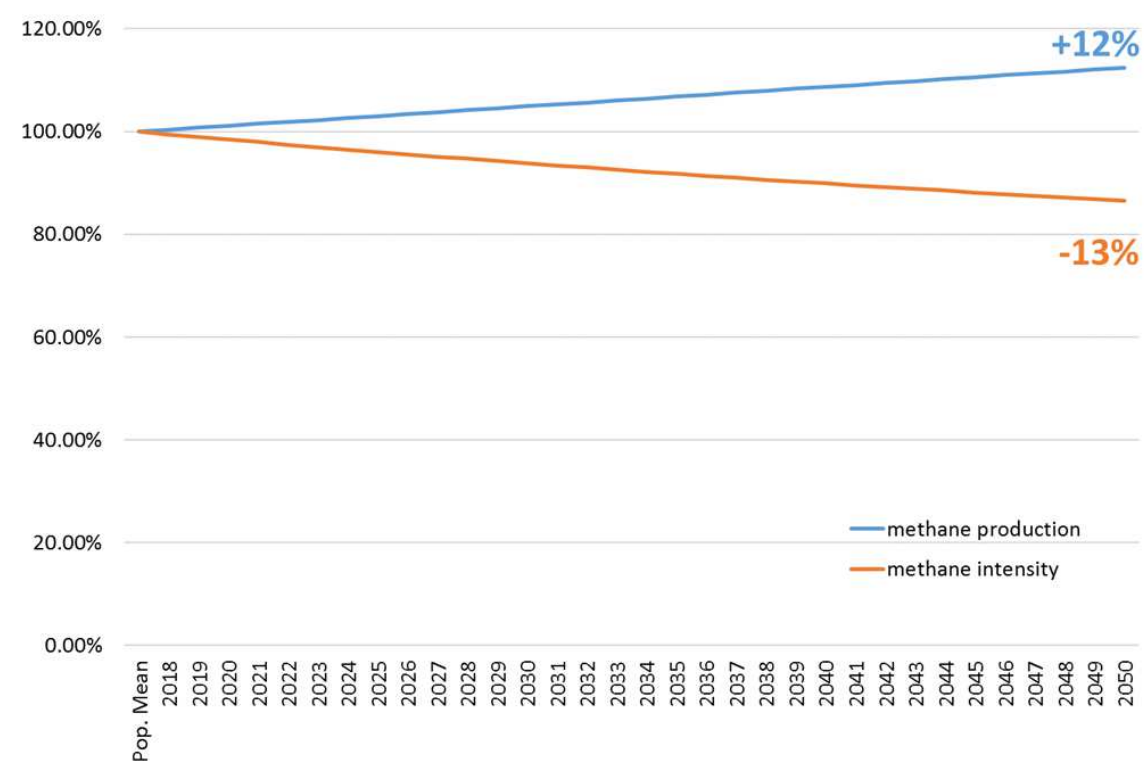

\section{Dairy - conclusion and discussion points}

- Effect of genetic progress through correlated responses:

- Methane production per animals increases

- Methane intensity decreases $=>$ effect of selection on production

- Dependent on correlations with other traits in national Dutch breeding goal (NVI)

- Still unsure what they are $=>$ need for individual recording 


\section{Conclusions and recommendations}

\section{General conclusions}

- Environmental impact of animal production decreases with $0.5-1.5 \%$ per year due to genetic progress on current breeding goals

- Methane intensity of dairy production

- GHG emissions and N and P efficiency of egg, broiler and pig production

\section{Recommendations}

- Account for individual variation in environmental impact traits

- For focussing on $\mathrm{N}$ and $\mathrm{P}$ efficiency, mineral contents in (edible parts of) the final product need to be monitored

- Additionally, account for human-edible output and input

- For dairy cows: methane measurements / predictions needed 


\section{References}

Bannink, A., M. W. Van Schijndel, and J. Dijkstra. 2011. A model of enteric fermentation in dairy cows to estimate methane emission for the Dutch National Inventory Report using the IPCC Tier 3 approach. Anim. Feed Sci. Technol. 166-167:603-618. doi: 10.1016/j.anifeedsci.2011.04.043

Caldas, J. 2015. Calorimetry and Body Composition Research in Broilers and Broiler Breeders, University of Arkansas, Arkansas.

CRV. 2017. CRV-Jaarstatistieken 2016 voor Nederland, CRV, Arnhem, the Netherlands.

DBEIS. 2017. Updated short-term traded carbon values used for modelling purposes, Department for Business, Energy and Industrial Strategy.

Ellen, E., T. Veldkamp, and Y. De Haas. s.a. Improving protein efficiency of livestock: pig and laying hen breeding as an example, Breed4Food, Wageningen, the Netherlands.

Finglas, P., M. Roe, H. Pinchen, R. Berry, S. Church, S. Dodhia, M. Farron-Wilson, and G. Swan. 2015. McCance and Widdowson's the Composition of Foods Integrated Dataset 2015 - User guide, PHE publications gateway number: 2014822. Public Health England, London, UK.

Gerber, P. J., H. Steinfeld, B. Henderson, A. Mottet, C. Opio, J. Dijkman, A. Falcucci, and G. Tempio. 2013. Tackling climate change through livestock - A global assessment of emissions and mitigation opportunities, Food and Agriculture Organization of the United Nations (FAO), Rome, Italy.

Groen, E. A., H. H. E. van Zanten, R. Heijungs, E. A. M. Bokkers, and I. J. M. de Boer. 2016. Sensitivity analysis of greenhouse gas emissions from a pork production chain. Journal of Cleaner Production 129:202-211. doi: 10.1016/j.jclepro.2016.04.081

Havenstein, G. B., P. R. Ferket, and M. A. Qureshi. 2003. Growth, livability, and feed conversion of 1957 versus 2001 broilers when fed representative 1957 and 2001 broiler diets. Poultry Science 82(10):1500-1508. doi: 10.1093/ps/82.10.1500

Hendrix Genetics. s.a. Parent Stock Management Guide (Version L7121-2), Hendrix Genetics, Boxmeer, the Netherlands.

ISA. s.a.-a. Dekalb White Product Guide - Alternative Production Systems (vs14.5), Institut de Sélection Animale BV.

ISA. s.a.-b. Isa Brown Product Guide - Alternative Production Systems (vs14.8), Institut de Sélection Animale BV, Boxmeer, the Netherlands.

Kool, A., J. Pluimers, and H. Blonk. 2014. Fossiel energiegebruik en broeikasgasemissies in de zuivelketen 1990-2012, Blonk Consultants, Gouda, the Netherlands.

KWIN. 2011. Kwantitatieve Informatie Veehouderij 2011-2012. Wageningen Livestock Research, Wageningen, the Netherlands.

KWIN. 2013. Kwantitatieve Informatie Veehouderij 2013-2014. Wageningen Livestock Research, Wageningen, the Netherlands.

KWIN. 2017. Kwantitatieve Informatie Veehouderij 2017-2018. Wageningen Livestock Research, Wageningen, the Netherlands.

Lassen, J., and P. Lovendahl. 2016. Heritability estimates for enteric methane emissions from Holstein cattle measured using noninvasive methods. J. Dairy Sci. 99(3):1959-1967. doi: $10.3168 /$ jds.2015-10012

Lassen, J., N. A. Poulsen, M. K. Larsen, and A. J. Buitenhuis. 2016. Genetic and genomic relationship between methane production measured in breath and fatty acid content in milk samples from Danish Holsteins. Animal Production Science 56(3)doi: 10.1071/an15489

Niu, M., E. Kebreab, A. N. Hristov, J. Oh, C. Arndt, A. Bannink, A. R. Bayat, A. F. Brito, T. Boland, D. Casper, L. A. Crompton, J. Dijkstra, M. A. Eugene, P. C. Garnsworthy, M. N. Haque, A. L. F. Hellwing, P. Huhtanen, M. Kreuzer, B. Kuhla, P. Lund, J. Madsen, C. Martin, S. C. McClelland, M. McGee, P. J. Moate, S. Muetzel, C. Munoz, P. O'Kiely, N. Peiren, C. K. Reynolds, A. Schwarm, K. J. Shingfield, T. M. Storlien, M. R. Weisbjerg, D. R. Yanez-Ruiz, and Z. Yu. 2018. Prediction of enteric methane production, yield, and intensity in dairy cattle using an intercontinental database. Global Change Biol. 24(8):3368-3389. doi: 10.1111/gcb.14094 
Pelletier, N. 2018. Changes in the life cycle environmental footprint of egg production in Canada from 1962 to 2012. Journal of Cleaner Production 176:1144-1153. doi: 10.1016/j.jclepro.2017.11.212

Pelletier, N., M. Ibarburu, and H. Xin. 2014. Comparison of the environmental footprint of the egg industry in the United States in 1960 and 2010. Poult Sci 93(2):241-255. doi: 10.3382/ps.201303390

Pettey, L. A., G. L. Cromwell, Y. D. Jang, and M. D. Lindemann. 2015. Estimation of calcium and phosphorus content in growing and finishing pigs: Whole empty body components and relative accretion rates. J. Anim. Sci. 93(1):158-167. doi: 10.2527/jas.2014-7602

Sevillano, C. A., C. V. Nicolaiciuc, F. Molist, J. Pijlman, and R. Bergsma. 2018. Effect of feeding cereals-alternative ingredients diets or corn-soybean meal diets on performance and carcass characteristics of growing-finishing gilts and boars. J. Anim. Sci. 96(11):4780-4788. doi: 10.1093/jas/sky339

Tallentire, C. W., I. Leinonen, and I. Kyriazakis. 2018. Artificial selection for improved energy efficiency is reaching its limits in broiler chickens. Scientific Reports 8(1):1168. doi: 10.1038/s41598-018-19231-2

Van Winkoop, C. 2013. The contribution of layer chicken breeding to the reduction of climate change. MSc, Wageningen University, Wageningen, the Netherlands.

Vellinga, T. V., H. Blonk, M. Marinussen, W. J. Van Zeist, I. J. M. De Boer, and D. Starmans. 2013. Methodology used in FeedPrint: a tool quantifying greenhouse gas emissions of feed production and utilization. Report 674, Wageningen UR Livestock Research, Wageningen, the Netherlands. http://edepot.wur.nl/254098

Wageningen Livestock Research. 2015. FeedPrint 2015.03. Wageningen UR, Wageningen, the Netherlands.

Wageningen Livestock Research. 2018. FeedPrint 2018.01. Wageningen UR, Wageningen, the Netherlands. 


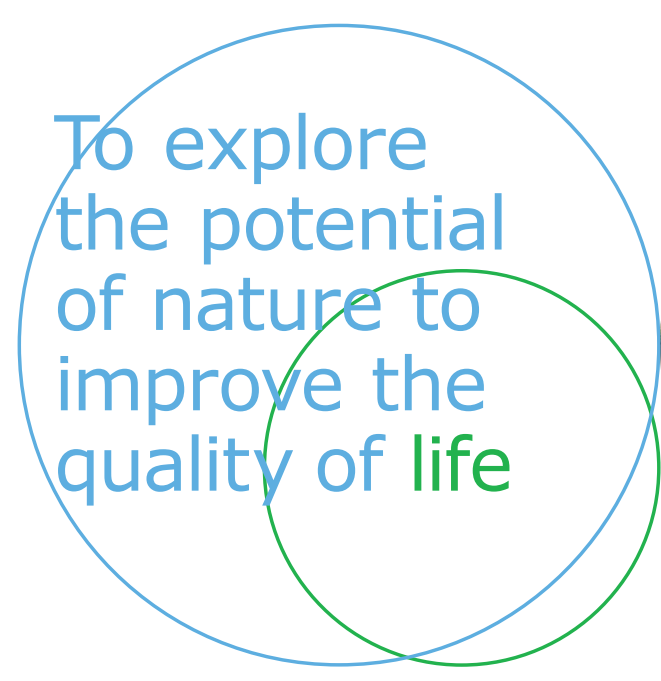

Wageningen Livestock Research

\section{P.O. Box 338}

$6700 \mathrm{AH}$ Wageningen

The Netherlands

$\mathrm{T}+31(0) 317483953$

E info.livestockresearch@wur.nl www.wur.nl/livestock-research

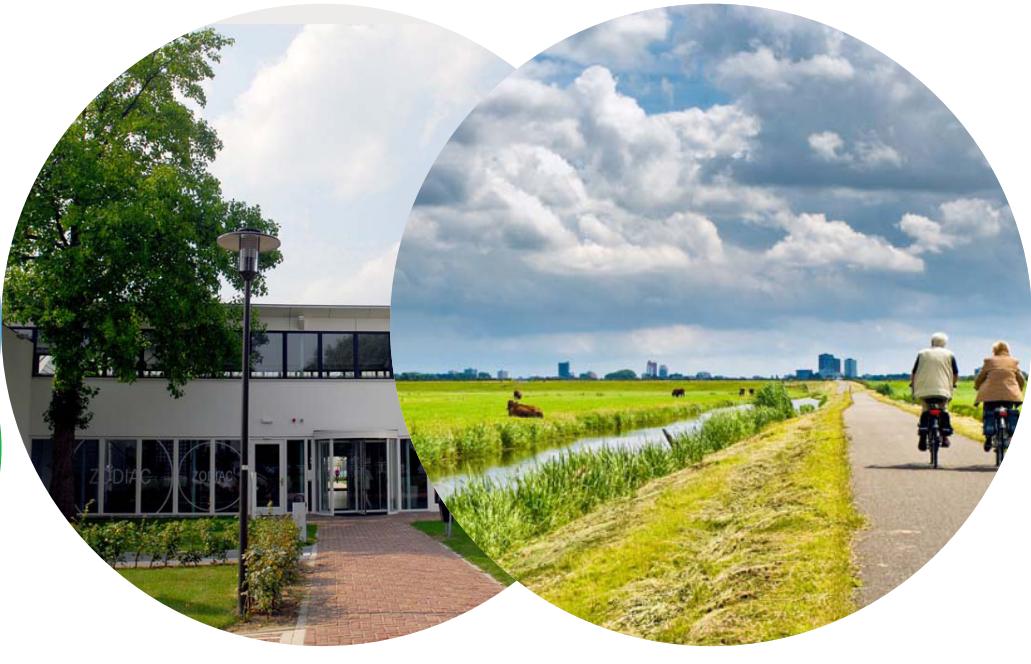

Wageningen Livestock Research creates science based solutions for a sustainable and profitable livestock sector. Together with our clients, we integrate scientific knowledge and practical experience to develop livestock concepts for future generations.

Wageningen Livestock Research is part of Wageningen University \& Research. Together we work on the mission: 'To explore the potential of nature to improve the quality of life'. A staff of 6,500 and 10,000 students from over 100 countries are working worldwide in the domain of healthy food and living environment for governments and the business community-at-large. The strength of Wageningen University \& Research lies in its ability to join the forces of specialised research institutes and the university. It also lies in the combined efforts of the various fields of natural and social sciences. This union of expertise leads to scientific breakthroughs that can quickly be put into practice and be incorporated into education. This is the Wageningen Approach. 